CUBO A Mathematical Journal Vol.13, $N^{\underline{O}}$ 03, (91-115). October 2011

\title{
Uncertainty principle for the Riemann-Liouville operator
}

\author{
HLeili Khaled \\ Faculty of Applied Mathematics,Département de Mathématiques et d'Informatique, \\ Institut national des sciences appliquées et de Thechnologie, \\ Centre Urbain Nord BP 676 - 1080 Tunis cedex, Tunisia, \\ email: khaled.hleili@gmail.com \\ OMRI SLIM \\ Département de Mathématiques Appliquées, \\ Institut préparatoire aux études d'ingénieurs, \\ Campus universitaire Mrezka - 8000 Nabeul, Tunisia. \\ email: slim.omri@issig.rnu.tn \\ and \\ LAKHDAR T. RACHDI \\ Département de Mathématiques, \\ Faculté des Sciences de Tunis, \\ 2092 El Manar II, Tunisia. \\ email: lakhdartannech.rachdi@fst.rnu.tn
}

\begin{abstract}
A Beurling-Hörmander theorem's is proved for the Fourier transform connected with the Riemann-Liouville operator. Nextly, Gelfand-Shilov and Cowling-Price type theorems are established.

\section{RESUMEN}

Se demuestra el teorema de Beurling-Hörmander por la transformada de Fourier conectada con el operador de Riemann-Liouville. Además, se establecen teoremas tipo de Gelfand-Shilov y Cowling-Price.
\end{abstract}


Keywords: Beurling-Hörmander theorem, Gelfand-Shilov theorem, Cowling- Price theorem, Fourier transform, Riemann-Liouville operator.

Mathematics Subject Classification: 43A32; 42B10.

\section{Introduction}

The uncertainty principles play an important role in harmonic analysis and have been studied by many authors, and from many points of view [12, 15. These principles state that a function $f$ and its Fourier transform $\widehat{f}$ cannot be simultaneously sharply localized. Theorems of Hardy, Morgan, Gelfand-Shilov, or Cowlong-Price,... are established for several Fourier transforms [8, 14, 19, 20, 21], the most recent being the well known Beurling-Hörmander theorem's which has been proved by Hörmander [16], who took an idea of Beurling [4. This theorem states that if $f$ is an integrable function on $\mathbb{R}$ with respect to the Lebesgue measure, and if

$$
\iint_{\mathbb{R}^{2}}|f(x)||\widehat{f}(y)| e^{|x y|} \mathrm{d} x \mathrm{~d} y<+\infty
$$

then $f=0$ almost everywhere.

Later, Bonami, Demange and Jaming [5] have generalized the above theorem and have established a strong multidimensional version of this uncertainty principle [15, by showing the following result if $f$ is a square integrable function on $\mathbb{R}^{n}$ with respect to the Lebesgue measure, then

$$
\int_{\mathbb{R}^{n}} \int_{\mathbb{R}^{n}} \frac{|f(x)||\widehat{f}(y)|}{(1+|x|+|y|)^{d}} e^{|\langle x / y\rangle|} d x d y<+\infty,
$$

if and only if $f$ may be written as

$$
f(x)=P(x) e^{-\langle A x / x\rangle}
$$

where $A$ is a real positive definite symmetric matrix and $P$ is a polynomial with $\operatorname{degree}(P)<\frac{d-n}{2}$. In particular for $\mathrm{d} \leqslant \mathrm{n}, \mathrm{f}$ is identically zero.

The Beurling-Hörmander uncertainty principle in its weak and strong forms has been studied by many authors, and for various Fourier transforms. In particular, Bouattour and Trimèche [6] have showed this theorem for the hypergroup of Chébli-Trimèche, Kamoun and Trimèche [17] have proved an analogue of the Beurling-Hörmander theorem for some singular partial differential operators, Trimèche 22] has showed this uncertainty principle for the Dunkl transform, we cite also Yakubovich [26], who has established the same result for the Kontorovich-Lebedev transform.

The Beurling-Hörmander uncertainty principle implies many other known quantitative uncertainty principles as those of Gelfand-Shilov [13, Cowling-Price [8], Morgan [3, 19] or also the one of Hardy 14.

In 2], the third author with the others have considered the singular partial differential oper- 
ators defined by

$$
\left\{\begin{array}{l}
\Delta_{1}=\frac{\partial}{\partial x} \\
\left.\Delta_{2}=\frac{\partial^{2}}{\partial r^{2}}+\frac{2 \alpha+1}{r} \frac{\partial}{\partial r}-\frac{\partial^{2}}{\partial x^{2}} ;(r, x) \in\right] 0,+\infty[\times \mathbb{R} ; \alpha \geq 0,
\end{array}\right.
$$

and they associated to $\Delta_{1}$ and $\Delta_{2}$ the following integral transform, called the Riemann-Liouville operator which is defined on $\mathscr{C}_{*}\left(\mathbb{R}^{2}\right.$ ) (The space of continuous functions on $\mathbb{R}^{2}$, even with respect to he first variable) by

$\mathscr{R}_{\alpha}(f)(r, x)= \begin{cases}\frac{\alpha}{\pi} \int_{-1}^{1} \int_{-1}^{1} f\left(r s \sqrt{1-t^{2}}, x+r t\right)\left(1-t^{2}\right)^{\alpha-\frac{1}{2}}\left(1-s^{2}\right)^{\alpha-1} d t d s, & \text { if } \alpha>0, \\ \frac{1}{\pi} \int_{-1}^{1} f\left(r \sqrt{1-t^{2}}, x+r t\right) \frac{d t}{\sqrt{\left(1-t^{2}\right)}} ; & \text { if } \alpha=0 .\end{cases}$

The Fourier transform connected with the operator $\mathscr{R}_{\alpha}$ is defined by

$$
\mathscr{F}_{\alpha}(f)(\mu, \lambda)=\int_{0}^{+\infty} \int_{\mathbb{R}} f(r, x) \varphi_{\mu, \lambda}(r, x) d v_{\alpha}(r, x),
$$

where

$\varphi_{\mu, \lambda}(r, x)=\mathscr{R}_{\alpha}\left(\cos (\mu.) e^{-i \lambda .}\right)(r, x)$.

$d v_{\alpha}$ is the measure defined on $[0,+\infty[\times \mathbb{R}$ by,

$$
d v_{\alpha}(r, x)=\frac{r^{2 \alpha+1}}{2^{\alpha} \Gamma(\alpha+1) \sqrt{2 \pi}} d r \otimes d x
$$

Many harmonic analysis results are established for the Fourier transform $\mathscr{F}_{\alpha}$ (Inversion formula, Plancherel's formula, Paley-Winer and Plancherel's theorems...).

The aim of this work is to establish the Beurling-Hörmander theorem for the fourier transform $\mathscr{F}_{\alpha}$ and to deduce the analogues of the Gelfand-Shilov and the Cowling-Price theorems for this transform.

More precisely, in the second section, we give some basic harmonic analysis results related to the Fourier transform $\mathscr{F}_{\alpha}$. The third section is devoted to establish the main result of this paper, that is the the Beurling-Hörmander theorem 
- Let $f$ be a square integrable function on $\left[0,+\infty\left[\times \mathbb{R}\right.\right.$ with respect to the measure $d v_{\alpha}$. Let $d$ be a real number, $d \geqslant 0$. If

$$
\iint_{\Gamma_{+}} \int_{0}^{+\infty} \int_{\mathbb{R}} \frac{|f(r, x)|\left|\mathscr{F}_{\alpha}(f)(\mu, \lambda)\right|}{(1+|(r, x)|+|\theta(\mu, \lambda)|)^{d}} e^{|(r, x)||\theta(\mu, \lambda)|} d v_{\alpha}(r, x) d \widetilde{\gamma}_{\alpha}(\mu, \lambda)<+\infty
$$

Then

i) For $d \leqslant 2, f=0$.

ii) For $d>2$, there exist a positive constant $a$ and a polynomial $P$ on $\mathbb{R}^{2}$ even with respect to the first variable, such that

$$
f(r, x)=P(r, x) e^{-a\left(r^{2}+x^{2}\right)},
$$

with $\operatorname{degree}(P)<\frac{d}{2}-1$,

where

$\Gamma_{+}=[0,+\infty[\times \mathbb{R} \cup\{(i t, x) \mid(t, x) \in[0,+\infty[\times \mathbb{R}, t \leqslant|x|\}$.

$\theta$ is the function defined on the set $\Gamma_{+}$by

$$
\theta(\mu, \lambda)=\left(\sqrt{\mu^{2}+\lambda^{2}}, \lambda\right) .
$$

$\mathrm{d} \widetilde{\gamma}_{\alpha}$ the measure defined on the set $\Gamma_{+}$by

$$
\begin{aligned}
\iint_{\Gamma_{+}} g(\mu, \lambda) d \widetilde{\gamma}_{\alpha}(\mu, \lambda) & =\frac{1}{\pi}\left(\int_{0}^{+\infty} \int_{\mathbb{R}} g(\mu, \lambda)\left(\mu^{2}+\lambda^{2}\right)^{-\frac{1}{2}} \mu d \mu d \lambda\right. \\
& \left.+\int_{\mathbb{R}} \int_{0}^{|\lambda|} g(i \mu, \lambda)\left(\lambda^{2}-\mu^{2}\right)^{-\frac{1}{2}} \mu d \mu d \lambda\right) .
\end{aligned}
$$

The last section of this paper contains the following results that are respectively the Gelfand-Shilov and the Cowling-Price theorems for $\mathscr{F}_{\alpha}$

- Let $p, q$ be two conjugate exponents, $p, q \in] 1,+\infty[$. Let $d, \xi, \eta$ be non negative real numbers such that $\xi \eta \geqslant 1$. Let $f$ be a measurable function on $\mathbb{R}^{2}$, even with respect to the first variable, such that $f \in L^{2}\left(d v_{\alpha}\right)$. If

$$
\int_{0}^{+\infty} \int_{\mathbb{R}} \frac{|f(r, x)| e^{\frac{\xi^{p}|(r, x)| p}{p}}}{(1+|(r, x)|)^{d}} d v_{\alpha}(r, x)<+\infty
$$

and

$$
\iint_{\Gamma_{+}} \frac{\left|\mathscr{F}_{\alpha}(f)(\mu, \lambda)\right| e^{\frac{\eta^{q}|\theta(\mu, \lambda)| q}{q}}}{(1+|\theta(\mu, \lambda)|)^{\mathrm{d}}} \mathrm{d} \widetilde{\gamma}_{\alpha}(\mu, \lambda)<+\infty
$$

then

i) For $d \leqslant 1, f=0$.

$2 i)$ For $d>1$, we have 
a) $f=0$ for $\xi \eta>1$.

b) $f=0$ for $\xi \eta=1$, and $p \neq 2$.

c) $f(r, x)=P(r, x) e^{-a\left(r^{2}+x^{2}\right)}$, for $\xi \eta=1$, and $p=q=2$,

where $\mathrm{a}>0$, and $\mathrm{P}$ is a polynomial on $\mathbb{R}^{2}$ even with respect to the first variable, with degree $(\mathrm{P})<$ $\mathrm{d}-1$.

- Let $\xi, \eta, \omega_{1}, \omega_{2}$ be non negative real numbers such that $\xi \eta \geqslant \frac{1}{4}$. Let $p, q$ be two exponents, $p, q \in[1,+\infty]$, and let $f$ be a measurable function on $\mathbb{R}^{2}$, even with respect to the first variable such that $f \in \mathrm{L}^{2}\left(\mathrm{~d} v_{\alpha}\right)$. If

$$
\left\|\frac{e^{\xi|(. . .)|^{2}}}{(1+|(., .)|)^{\omega_{1}}} f\right\|_{p, v_{\alpha}}<+\infty
$$

and

$$
\left\|\frac{e^{\eta|\theta(. . .)|^{2}}}{(1+|\theta(., .)|)^{\omega_{2}}} \mathscr{F}_{\alpha}(f)\right\|_{\mathbf{q}, \tilde{\gamma}_{\alpha}}<+\infty
$$

then

i) For $\xi \eta>\frac{1}{4}, f=0$.

ii) For $\xi \eta=\frac{1}{4}$, there exist a positive constant a and a polynomial $\mathrm{P}$ on $\mathbb{R}^{2}$, even with respect to the first variable, such that

$$
f(r, x)=P(r, x) e^{-a\left(r^{2}+x^{2}\right)} .
$$

\section{The Fourier transform associated with the Riemann-Liouville operator}

It's well known 2] that for all $(\mu, \lambda) \in \mathbb{C}^{2}$, the system

$$
\left\{\begin{array}{l}
\Delta_{1} u(r, x)=-i \lambda u(r, x), \\
\Delta_{2} u(r, x)=-\mu^{2} u(r, x), \\
u(0,0)=1, \frac{\partial u}{\partial r}(0, x)=0, \forall x \in \mathbb{R},
\end{array}\right.
$$

admits a unique solution $\varphi_{\mu, \lambda}$, given by

$$
\forall(r, x) \in \mathbb{R}^{2} ; \quad \varphi_{\mu, \lambda}(r, x)=j_{\alpha}\left(r \sqrt{\mu^{2}+\lambda^{2}}\right) e^{-i \lambda x},
$$

where

$$
j_{\alpha}(z)=\frac{2^{\alpha} \Gamma(\alpha+1)}{z^{\alpha}} J_{\alpha}(z)=\Gamma(\alpha+1) \sum_{n=0}^{+\infty} \frac{(-1)^{n}}{n ! \Gamma(\alpha+n+1)}\left(\frac{z}{2}\right)^{2 n}, \quad z \in \mathbb{C},
$$


and $\mathrm{J}_{\alpha}$ is the Bessel function of the first kind and index $\alpha$ [9, 10, 18, 25].

The modified Bessel function $j_{\alpha}$ has the following integral representation [18, 25], for all $z \in \mathbb{C}$, we have

$$
j_{\alpha}(z)= \begin{cases}\frac{2 \Gamma(\alpha+1)}{\sqrt{\pi} \Gamma\left(\alpha+\frac{1}{2}\right)} \int_{0}^{1}\left(1-t^{2}\right)^{\alpha-\frac{1}{2}} \cos (z t) d t, & \text { if } \alpha>-\frac{1}{2} \\ \cos (z), & \text { if } \alpha=-\frac{1}{2} .\end{cases}
$$

From the relation (2.2), we deduce that for all $z \in \mathbb{C}$, we have

$$
\left|j_{\alpha}(z)\right| \leqslant e^{|\operatorname{Im}(z)|} .
$$

From the properties of the modified Bessel function $j_{\alpha}$, we deduce that the eigenfunction $\varphi_{\mu, \lambda}$ satisfies the following properties

$$
\sup _{(r, x) \in \mathbb{R}^{2}}\left|\varphi_{\mu, \lambda}(r, x)\right|=1,
$$

if and only if $(\mu, \lambda)$ belongs to the set

$$
\Gamma=\mathbb{R}^{2} \cup\left\{(i t, x)\left|(t, x) \in \mathbb{R}^{2},\right| t|\leq| x \mid\right\} .
$$

The eigenfunction $\varphi_{\mu, \lambda}$ has the following Mehler integral representation

$$
\varphi_{\mu, \lambda}(r, x)= \begin{cases}\frac{\alpha}{\pi} \int_{-1}^{1} \int_{-1}^{1} \cos \left(\mu r s \sqrt{1-t^{2}}\right) e^{-i \lambda(x+r t)}\left(1-t^{2}\right)^{\alpha-\frac{1}{2}}\left(1-s^{2}\right)^{\alpha-1} d t d s ; & \text { if } \alpha>0, \\ \frac{1}{\pi} \int_{-1}^{1} \cos \left(r \mu \sqrt{1-t^{2}}\right) e^{-i \lambda(x+r t)} \frac{d t}{\sqrt{1-t^{2}}} ; & \text { if } \alpha=0 .\end{cases}
$$

This integral representation allows to define the so-called Riemann-Liouville operator associated with $\Delta_{1}, \Delta_{2}$ by

$$
\mathscr{R}_{\alpha}(f)(r, x)= \begin{cases}\frac{\alpha}{\pi} \int_{-1}^{1} \int_{-1}^{1} f\left(r s \sqrt{1-t^{2}}, x+r t\right)\left(1-t^{2}\right)^{\alpha-\frac{1}{2}}\left(1-s^{2}\right)^{\alpha-1} d t d s ; & \text { if } \alpha>0, \\ \frac{1}{\pi} \int_{-1}^{1} f\left(r \sqrt{1-t^{2}}, x+r t\right) \frac{d t}{\sqrt{\left(1-t^{2}\right)}} ; & \text { if } \alpha=0 .\end{cases}
$$

where $\mathrm{f}$ is a continuous function on $\mathbb{R}^{2}$, even with respect to the first variable.

The transform $\mathscr{R}_{\alpha}$ generalizes the "mean operator" defined by

$$
\mathscr{R}_{0}(f)(r, x)=\frac{1}{2 \pi} \int_{0}^{2 \pi} f(r \sin \theta, x+r \cos \theta) d \theta .
$$

In the following, we denote by 
$d m_{n+1}$ the measure defined on $\left[0,+\infty\left[\times \mathbb{R}^{n}\right.\right.$ by,

$$
d m_{n+1}(r, x)=\sqrt{\frac{2}{\pi}} \frac{1}{(2 \pi)^{\frac{n}{2}}} d r \otimes d x .
$$

$L^{p}\left(d m_{n+1}\right)$ the space of measurable functions $f$ on $\left[0,+\infty\left[\times \mathbb{R}^{n}\right.\right.$, such that

$$
\begin{array}{ll}
\|f\|_{p, m_{n+1}}=\left(\int_{0}^{+\infty} \int_{\mathbb{R}^{n}}|f(r, x)|^{p} \mathrm{dm}_{n+1}(r, x)\right)^{\frac{1}{p}}<+\infty, & \text { if } p \in[1,+\infty[, \\
\|f\|_{\infty, m_{n+1}}=\operatorname{ess} \sup _{(r, x) \in\left[0,+\infty\left[\times \mathbb{R}^{n}\right.\right.}|f(r, x)|<+\infty, & \text { if } p=+\infty .
\end{array}
$$

$d v_{\alpha}$ the measure defined on $[0,+\infty[\times \mathbb{R}$, by

$$
d v_{\alpha}(r, x)=\frac{r^{2 \alpha+1}}{2^{\alpha} \Gamma(\alpha+1) \sqrt{2 \pi}} d r \otimes d x
$$

$L^{p}\left(d v_{\alpha}\right)$ the space of measurable functions $f$ on $\left[0,+\infty\left[\times \mathbb{R}\right.\right.$ such that $\|f\|_{p, v_{\alpha}}<+\infty$.

$\Gamma_{+}=[0,+\infty[\times \mathbb{R} \cup\{(i t, x) \mid(t, x) \in[0,+\infty[\times \mathbb{R}, t \leqslant|x|\}$.

$\mathscr{B}_{\Gamma_{+}}$the $\sigma$-algebra defined on $\Gamma_{+}$by

$$
\mathscr{B}_{\Gamma_{+}}=\left\{\theta^{-1}(\mathrm{~B}), \mathrm{B} \in \mathscr{B}([0,+\infty[\times \mathbb{R})\},\right.
$$

where $\theta$ is the bijective function defined on the set $\Gamma_{+}$by

$$
\theta(\mu, \lambda)=\left(\sqrt{\mu^{2}+\lambda^{2}}, \lambda\right) .
$$

d $\gamma_{\alpha}$ the measure defined on $\mathscr{B}_{\Gamma_{+}}$by

$$
\forall A \in \mathscr{B}_{\Gamma_{+}} ; \gamma_{\alpha}(A)=\nu_{\alpha}(\theta(A))
$$

$L^{p}\left(d \gamma_{\alpha}\right)$ the space of measurable functions $f$ on $\Gamma_{+}$, such that $\|f\|_{p, \gamma_{\alpha}}<+\infty$.

$\mathrm{d} \widetilde{\gamma}_{\alpha}$ the measure defined on $\mathscr{B}_{\Gamma_{+}}$by

$$
d \widetilde{\gamma}_{\alpha}(\mu, \lambda)=\frac{2^{\alpha+\frac{1}{2}} \Gamma(\alpha+1)}{\sqrt{\pi}\left(\mu^{2}+\lambda^{2}\right)^{\alpha+\frac{1}{2}}} d \gamma_{\alpha}(\mu, \lambda) .
$$

$S_{*}\left(\mathbb{R}^{2}\right)$ the Shwartz's space formed by the infinitely differentiable functions on $\mathbb{R}^{2}$, rapidly decreasing together with all their derivatives, and even with respect to the first variable.

Then we have the following properties. 
Proposition 2.1. i) For all non negative measurable function $\mathrm{g}$ on $\Gamma_{+}$, we have

$$
\begin{aligned}
\iint_{\Gamma_{+}} g(\mu, \lambda) d \gamma_{\alpha}(\mu, \lambda) & =\frac{1}{2^{\alpha} \Gamma(\alpha+1) \sqrt{2 \pi}}\left(\int_{0}^{+\infty} \int_{\mathbb{R}} g(\mu, \lambda)\left(\mu^{2}+\lambda^{2}\right)^{\alpha} \mu d \mu d \lambda\right. \\
& \left.+\int_{\mathbb{R}} \int_{0}^{|\lambda|} g(i \mu, \lambda)\left(\lambda^{2}-\mu^{2}\right)^{\alpha} \mu d \mu d \lambda\right) .
\end{aligned}
$$

ii) For all measurable function $\mathrm{f}$ on $\left[0,+\infty\left[\times \mathbb{R}\right.\right.$, the function fo $\theta$ is measurable on $\Gamma_{+}$. Furthermore if $\mathrm{f}$ is non negative or integrable function on $\left[0,+\infty\left[\times \mathbb{R}\right.\right.$ with respect to the measure $\mathrm{d} v_{\alpha}$, then we have

$$
\iint_{\Gamma_{+}}(f \circ \theta)(\mu, \lambda) d \gamma_{\alpha}(\mu, \lambda)=\int_{0}^{+\infty} \int_{\mathbb{R}} f(r, x) d v_{\alpha}(r, x) .
$$

iii) For all non negative measurable function $\mathrm{f}$, respectively integrable on $[0,+\infty[\times \mathbb{R}$ with respect to the measure $\mathrm{dm}_{2}$, we have

$$
\iint_{\Gamma_{+}}(f \circ \theta)(\mu, \lambda) d \widetilde{\gamma}_{\alpha}(\mu, \lambda)=\int_{0}^{+\infty} \int_{\mathbb{R}} f(r, x) d m_{2}(r, x) .
$$

In the following we shall define the Fourier transform $\mathscr{F}_{\alpha}$ associated with the operator $\mathscr{R}_{\alpha}$, and we shall give some properties that we use in the sequel.

Definition 2.1. The Fourier transform $\mathscr{F}_{\alpha}$ associated with the Riemann-Liouville operator $\mathscr{R}_{\alpha}$ is defined on $L^{1}\left(d v_{\alpha}\right)$ by

$$
\forall(\mu, \lambda) \in \Gamma ; \mathscr{F}_{\alpha}(f)(\mu, \lambda)=\int_{0}^{+\infty} \int_{\mathbb{R}} f(r, x) \varphi_{\mu, \lambda}(r, x) d v_{\alpha}(r, x) .
$$

Then, for all $(\mu, \lambda) \in \Gamma$,

$$
\mathscr{F}_{\alpha}(f)(\mu, \lambda)=\widetilde{\mathscr{F}}_{\alpha}(f) \circ \theta(\mu, \lambda),
$$

where for all $(\mu, \lambda) \in[0,+\infty[\times \mathbb{R}$,

$$
\widetilde{\mathscr{F}}_{\alpha}(f)(\mu, \lambda)=\int_{0}^{+\infty} \int_{\mathbb{R}} f(r, x) j_{\alpha}(r \mu) e^{-i \lambda x} d v_{\alpha}(r, x) .
$$

Moreover, the relation (2.4) implies that the Fourier transform $\mathscr{F}_{\alpha}$ is a bounded linear operator from $L^{1}\left(d v_{\alpha}\right)$ into $L^{\infty}\left(d \gamma_{\alpha}\right)$, and that for all $f \in L^{1}\left(d v_{\alpha}\right)$, we have

$$
\left\|\mathscr{F}_{\alpha}(f)\right\|_{\infty, \gamma_{\alpha}} \leqslant\|f\|_{1, v_{\alpha}} .
$$

Theorem 2.1 (Inversion formula). Let $\mathrm{f} \in \mathrm{L}^{1}\left(\mathrm{~d} v_{\alpha}\right)$ such that $\mathscr{F}_{\alpha}(\mathrm{f}) \in \mathrm{L}^{1}\left(\mathrm{~d} \gamma_{\alpha}\right)$, then for almost every $(\mathrm{r}, \mathrm{x}) \in[0,+\infty[\times \mathbb{R}$, we have

$$
\begin{aligned}
f(r, x) & =\iint_{\Gamma_{+}} \mathscr{F}_{\alpha}(f)(\mu, \lambda) \overline{\varphi_{\mu, \lambda}(r, x)} d \gamma_{\alpha}(\mu, \lambda) \\
& =\int_{0}^{+\infty} \int_{\mathbb{R}} \widetilde{\mathscr{F}}_{\alpha}(f)(\mu, \lambda) j_{\alpha}(r \mu) e^{i \lambda x} \mathrm{~d} v_{\alpha}(\mu, \lambda) .
\end{aligned}
$$


Lemma 2.2. Let $\mathfrak{R}_{\alpha}$ be the mapping defined for all non negative measurable function $\mathrm{g}$ on $[0,+\infty[\times \mathbb{R}$ by

$$
\begin{aligned}
\mathfrak{R}_{\alpha}(\mathrm{g})(\mathrm{r}, \mathrm{x}) & =\frac{2 \Gamma(\alpha+1)}{\sqrt{\pi} \Gamma\left(\alpha+\frac{1}{2}\right)} \int_{0}^{1}\left(1-\mathrm{s}^{2}\right)^{\alpha-\frac{1}{2}} \mathrm{~g}(\mathrm{rs}, \mathrm{x}) \mathrm{ds} \\
& =\frac{2 \Gamma(\alpha+1) \mathrm{r}^{-2 \alpha}}{\sqrt{\pi} \Gamma\left(\alpha+\frac{1}{2}\right)} \int_{0}^{r}\left(r^{2}-\mathrm{s}^{2}\right)^{\alpha-\frac{1}{2}} \mathrm{f}(\mathrm{s}, \mathrm{x}) \mathrm{ds}, \quad r>0 .
\end{aligned}
$$

Then for all non negative measurable functions $f, g$ on $[0,+\infty[\times \mathbb{R}$, we have

$$
\int_{0}^{+\infty} \int_{\mathbb{R}} f(r, x) \Re_{\alpha}(g)(r, x) d v_{\alpha}(r, x)=\int_{0}^{+\infty} \int_{\mathbb{R}} \mathscr{W}_{\alpha}(f)(r, x) g(r, x) d m_{2}(r, x),
$$

where $\mathscr{W}_{\alpha}$ is the classical Weyl transform defined for all non negative measurable function on $[0,+\infty[\times \mathbb{R}$ by

$$
\mathscr{W}_{\alpha}(f)(r, x)=\frac{1}{2^{\alpha+\frac{1}{2}} \Gamma\left(\alpha+\frac{1}{2}\right)} \int_{r}^{+\infty}\left(t^{2}-r^{2}\right)^{\alpha-\frac{1}{2}} f(t, x) 2 t d t .
$$

Proposition 2.2. For all $\mathrm{f} \in \mathrm{L}^{1}\left(\mathrm{~d} v_{\alpha}\right)$, the function $\mathscr{W}_{\alpha}(\mathrm{f})$ belongs to $\mathrm{L}^{1}\left(\mathrm{dm}_{2}\right)$, and we have

$$
\left\|\mathscr{W}_{\alpha}(f)\right\|_{1, m_{2}} \leqslant\|f\|_{1, v_{\alpha}} .
$$

Moreover, for all $(\mu, \lambda) \in[0,+\infty[\times \mathbb{R}$, we have

$$
\widetilde{\mathscr{F}}_{\alpha}(f)(\mu, \lambda)=\left(\Lambda_{2} \circ \mathscr{W}_{\alpha}\right)(f)(\mu, \lambda),
$$

where $\Lambda_{2}$ is the usual Fourier transform defined on $\mathrm{L}^{1}\left(\mathrm{dm}_{2}\right)$ by

$$
\Lambda_{2}(g)(\mu, \lambda)=\int_{0}^{+\infty} \int_{\mathbb{R}} g(r, x) \cos (r \mu) e^{-i \lambda x} d m_{2}(r, x) .
$$

Remark 2.1. It's well known [23, 24] that the transforms $\widetilde{\mathscr{F}_{\alpha}}$ and $\Lambda_{2}$ are topological isomorphisms from $S_{*}\left(\mathbb{R}^{2}\right)$ onto itself. Then by the relation (2.13), we deduce that the classical Weyl transform $\mathscr{W}_{\alpha}$ is also a topological isomorphism from $S_{*}\left(\mathbb{R}^{2}\right)$ onto itself.

Proposition 2.3. For all $\mathrm{f} \in \mathrm{S}_{*}\left(\mathbb{R}^{2}\right)$, we have

$$
\mathscr{W}_{\alpha}^{-1}(f)=(-1)^{1+\left[\alpha+\frac{1}{2}\right]} \mathscr{W}_{\left[\alpha+\frac{1}{2}\right]-\alpha+\frac{1}{2}}\left(\left(\frac{\partial}{\partial \mathrm{t}^{2}}\right)^{1+\left[\alpha+\frac{1}{2}\right]}(f)\right),
$$

where

$$
\left(\frac{\partial}{\partial t^{2}}\right)(f)(t, x)=\frac{1}{t} \frac{\partial f}{\partial t}(t, x)
$$

Proof. For $\sigma \in \mathbb{R}, \sigma>0$, let us define the so-called fractional transform $\mathscr{H}_{\sigma}$, defined on $\mathrm{S}_{*}\left(\mathbb{R}^{2}\right)$ by

$$
\mathscr{H}_{\sigma}(f)(r, x)=\frac{1}{2^{\sigma} \Gamma(\sigma)} \int_{r}^{+\infty}\left(t^{2}-r^{2}\right)^{\sigma-1} f(t, x) 2 t d t=\mathscr{W}_{\sigma-\frac{1}{2}}(f)(r, x) .
$$


From the remark 2.1, it follows that for all real number $\sigma>0$, the mapping $\mathscr{H}_{\sigma}$ is a topological isomorphism from $S_{*}\left(\mathbb{R}^{2}\right)$ onto itself.

Moreover, we have the following properties

For all $\sigma, \delta \in \mathbb{R} ; \quad \sigma, \delta>0$ and for every $f \in S_{*}\left(\mathbb{R}^{2}\right)$, we have

$$
\left(\mathscr{H}_{\sigma} \circ \mathscr{H}_{\delta}\right)(\mathrm{f})=\mathscr{H}_{\sigma+\delta}(\mathrm{f}) \text {. }
$$

For all $\sigma \in \mathbb{R}, \quad \sigma>0$, and for every integer $k$, we have

$$
\mathscr{H}_{\sigma}(\mathrm{f})=(-1)^{\mathrm{k}} \mathscr{H}_{\sigma+\mathrm{k}}\left(\left(\frac{\partial}{\partial \mathrm{t}^{2}}\right)^{\mathrm{k}}(\mathrm{f})\right) \text {. }
$$

where $\frac{\partial}{\partial t^{2}}$ is the linear continuous operator defined on $S_{*}\left(\mathbb{R}^{2}\right)$ by

$$
\frac{\partial}{\partial t^{2}}(f)(t, x)=\frac{1}{t} \frac{\partial f}{\partial t}(t, x) \text {. }
$$

The relation (2.15) allows us to extend the mapping $\mathscr{H}_{\sigma}$ on $\mathbb{R}$, by setting

$$
\mathscr{H}_{\sigma}(\mathrm{f})(\mathrm{r}, \mathrm{x})=(-1)^{\mathrm{k}} \mathscr{H}_{\sigma+k}\left(\left(\frac{\partial}{\partial \mathrm{t}^{2}}\right)^{\mathrm{k}}(\mathrm{f})\right),
$$

where $k$ is any integer such that $\sigma+k>0, \sigma \in \mathbb{R}$.

The extension $\mathscr{H}_{\sigma}, \sigma \in \mathbb{R}$ satisfies

$$
\left(\mathscr{H}_{\sigma} \circ \mathscr{H}_{\delta}\right)(\mathrm{f})=\mathscr{H}_{\sigma+\delta}(\mathrm{f}), \quad \sigma, \delta \in \mathbb{R}, \quad f \in S_{*}\left(\mathbb{R}^{2}\right),
$$

and $\mathscr{H}_{0}(\mathrm{f})=\mathrm{f}$, for all $\mathrm{f} \in \mathrm{S}_{*}\left(\mathbb{R}^{2}\right)$.

In particular, for all $\sigma \in \mathbb{R}$, the transform $\mathscr{H}_{\sigma}$ is a topological isomorphism from $S_{*}\left(\mathbb{R}^{2}\right)$ onto itself, and the isomorphism inverse is given by

$$
\mathscr{H}_{\sigma}^{-1}=\mathscr{H}_{-\sigma}
$$

Thus, for all real number $\sigma$, we have

$$
\mathscr{H}_{\sigma}^{-1}(f)=(-1)^{1+[\sigma]} \mathscr{H}_{1+[\sigma]-\sigma}\left(\left(\frac{\partial}{\partial t^{2}}\right)^{1+[\sigma]}(f)\right) .
$$

In particular

$$
\mathscr{W}_{\alpha}^{-1}(f)=\mathscr{H}_{\alpha+\frac{1}{2}}^{-1}(f)=(-1)^{1+\left[\alpha+\frac{1}{2}\right]} \mathscr{H}_{\left[\alpha+\frac{1}{2}\right]-\alpha+\frac{1}{2}}\left(\left(\frac{\partial}{\partial t^{2}}\right)^{1+\left[\alpha+\frac{1}{2}\right]}(f)\right) .
$$

\section{The Beurling-Hörmander theorem for the Riemann-Liouville operator}

In this section, we shall establish the main result of this paper, that is the Beurling-Hörmander theorem for the Fourier transform $\mathscr{F}_{\alpha}$.

We recall firstly the following result that has been established by Bonami, Demange and Jaming [5]. 
Theorem 3.1. Let $\mathrm{f}$ be a measurable function on $\mathbb{R} \times \mathbb{R}^{n}$, even with respect to the first variable such that $\mathrm{f} \in \mathrm{L}^{2}\left(\mathrm{dm}_{\mathrm{n}+1}\right)$, and let $\mathrm{d}$ be a real number, $\mathrm{d} \geq 0$. If

$$
\int_{0}^{+\infty} \int_{\mathbb{R}^{n}} \int_{0}^{+\infty} \int_{\mathbb{R}^{n}} \frac{|f(r, x)|\left|\Lambda_{n+1}(f)(s, y)\right|}{(1+|(r, x)|+|(s, y)|)^{d}} e^{|(r, x)||(s, y)|} \mathrm{dm}_{n+1}(r, x) d m_{n+1}(s, y)<+\infty,
$$

then there exist a positive constant $\mathrm{a}$ and a polynomial $\mathrm{P}$ on $\mathbb{R} \times \mathbb{R}^{n}$, even with respect to the first variable, such that

$$
f(r, x)=P(r, x) e^{-a\left(r^{2}+|x|^{2}\right)},
$$

with $\operatorname{degree}(\mathrm{P})<\frac{\mathrm{d}-(\mathrm{n}+1)}{2}$.

In the following, we will establish some intermediary results that we use nextly.

Lemma 3.2. Let $\mathrm{f} \in \mathrm{L}^{2}\left(\mathrm{~d} v_{\alpha}\right)$ such that

$$
\iint_{\Gamma_{+}} \int_{0}^{+\infty} \int_{\mathbb{R}} \frac{|f(r, x)|\left|\mathscr{F}_{\alpha}(f)(\mu, \lambda)\right|}{(1+|(r, x)|+|\theta(\mu, \lambda)|)^{d}} e^{|(r, x)||\theta(\mu, \lambda)|} \mathrm{d} v_{\alpha}(r, x) d \widetilde{\gamma}_{\alpha}(\mu, \lambda)<+\infty,
$$

then the function $\mathrm{f}$ belongs to the space $\mathrm{L}^{1}\left(\mathrm{~d} v_{\alpha}\right)$.

Proof. From the hypothesis, and the relations (2.5) and (2.6), we have

$$
\begin{aligned}
& \iint_{\Gamma_{+}} \int_{0}^{+\infty} \int_{\mathbb{R}} \frac{|f(r, x)|\left|\mathscr{F}_{\alpha}(f)(\mu, \lambda)\right|}{(1+|(r, x)|+|\theta(\mu, \lambda)|)^{\mathrm{d}}} e^{|(r, x)||\theta(\mu, \lambda)|} \mathrm{d} v_{\alpha}(r, x) \mathrm{d} \widetilde{\gamma}_{\alpha}(\mu, \lambda) \\
& \quad=\int_{0}^{+\infty} \int_{\mathbb{R}} \int_{0}^{+\infty} \int_{\mathbb{R}} \frac{|f(r, x)|\left|\widetilde{F}_{\alpha}(f)(\mu, \lambda)\right|}{(1+|(r, x)|+|(\mu, \lambda)|)^{\mathrm{d}}} e^{|(r, x)||(\mu, \lambda)|} \mathrm{d} v_{\alpha}(r, x) \mathrm{dm}_{2}(\mu, \lambda)<+\infty .
\end{aligned}
$$

We assume of course that $f \neq 0$. Then, there exists $\left(\mu_{0}, \lambda_{0}\right) \in\left[0,+\infty\left[\times \mathbb{R}\right.\right.$, such that $\left(\mu_{0}, \lambda_{0}\right) \neq$ $(0,0), \widetilde{\mathscr{F}}_{\alpha}(f)\left(\mu_{0}, \lambda_{0}\right) \neq 0$, and

$$
\left|\widetilde{\mathscr{F}}_{\alpha}(f)\left(\mu_{0}, \lambda_{0}\right)\right| \int_{0}^{+\infty} \int_{\mathbb{R}}|f(r, x)| \frac{e^{|(r, x)|\left|\left(\mu_{0}, \lambda_{0}\right)\right|}}{\left(1+|(r, x)|+\left|\left(\mu_{0}, \lambda_{0}\right)\right|\right)^{d}} d v_{\alpha}(r, x)<+\infty,
$$

hence

$$
\int_{0}^{+\infty} \int_{\mathbb{R}}|f(r, x)| \frac{e^{|(r, x)|\left|\left(\mu_{0}, \lambda_{0}\right)\right|}}{\left(1+|(r, x)|+\left|\left(\mu_{0}, \lambda_{0}\right)\right|\right)^{d}} d v_{\alpha}(r, x)<+\infty .
$$

Let $h$ be the function defined on $[0,+\infty[$ by

$$
h(s)=\frac{e^{s\left|\left(\mu_{0}, \lambda_{0}\right)\right|}}{\left(1+s+\left|\left(\mu_{0}, \lambda_{0}\right)\right|\right)^{d}},
$$

then the function $\mathrm{h}$ admits a minimum attained at

$$
s_{0}= \begin{cases}\frac{d}{\left|\left(\mu_{0}, \lambda_{0}\right)\right|}-1-\left|\left(\mu_{0}, \lambda_{0}\right)\right|, & \text { if } \frac{d}{\left|\left(\mu_{0}, \lambda_{0}\right)\right|}>1+\left|\left(\mu_{0}, \lambda_{0}\right)\right| ; \\ 0, & \text { if } \frac{d}{\left|\left(\mu_{0}, \lambda_{0}\right)\right|} \leqslant 1+\left|\left(\mu_{0}, \lambda_{0}\right)\right| .\end{cases}
$$


Consequently,

$$
\begin{aligned}
\int_{0}^{+\infty} \int_{\mathbb{R}}|f(r, x)| d v_{\alpha}(r, x) & \leqslant \frac{1}{h\left(s_{0}\right)} \int_{0}^{+\infty} \int_{\mathbb{R}} \frac{|f(r, x)| e^{|(r, x)|\left|\left(\mu_{0}, \lambda_{0}\right)\right|}}{\left(1+|(r, x)|+\left|\left(\mu_{0}, \lambda_{0}\right)\right|\right)} d v_{\alpha}(r, x) \\
& <+\infty
\end{aligned}
$$

Lemma 3.3. Let $\mathrm{f} \in \mathrm{L}^{2}\left(\mathrm{~d} v_{\alpha}\right)$ such that

$$
\iint_{\Gamma_{+}} \int_{0}^{+\infty} \int_{\mathbb{R}} \frac{|f(r, x)|\left|\mathscr{F}_{\alpha}(f)(\mu, \lambda)\right|}{(1+|(r, x)|+|\theta(\mu, \lambda)|)^{d}} e^{|(r, x)||\theta(\mu, \lambda)|} d v_{\alpha}(r, x) d \widetilde{\gamma}_{\alpha}(\mu, \lambda)<+\infty
$$

Then, there exists a $>0$ such that the function $\widetilde{\mathscr{F}}_{\alpha}(\mathrm{f})$ is analytic on the set

$$
\mathrm{B}_{\mathrm{a}}=\left\{(\mu, \lambda) \in \mathbb{C}^{2}|| \operatorname{Im}(\mu)|<\mathrm{a},| \operatorname{Im}(\lambda) \mid<\mathrm{a}\right\} .
$$

Proof. From the proof of the lemma 3.2 , there exists $\left(\mu_{0}, \lambda_{0}\right) \neq(0,0)$, such that

$$
\int_{0}^{+\infty} \int_{\mathbb{R}} \frac{|f(r, x)| e^{|(r, x)|\left|\left(\mu_{0}, \lambda_{0}\right)\right|}}{\left(1+|(r, x)|+\left|\left(\mu_{0}, \lambda_{0}\right)\right|\right)^{d}} d v_{\alpha}(r, x)<+\infty
$$

Let $a>0$, such that $0<2 a<\left|\left(\mu_{0}, \lambda_{0}\right)\right|$. Then we have

$$
\begin{aligned}
\int_{0}^{+\infty} & \int_{\mathbb{R}} \frac{|f(r, x)| e^{|(r, x)|\left|\left(\mu_{0}, \lambda_{0}\right)\right|}}{\left(1+|(r, x)|+\left|\left(\mu_{0}, \lambda_{0}\right)\right|\right)^{d}} d v_{\alpha}(r, x) \\
\quad= & \int_{0}^{+\infty} \int_{\mathbb{R}}|f(r, x)| e^{2 a|(r, x)|} \frac{e^{|(r, x)|\left(\left|\left(\mu_{0}, \lambda_{0}\right)\right|-2 a\right)}}{\left(1+|(r, x)|+\left|\left(\mu_{0}, \lambda_{0}\right)\right|\right)^{d}} d v_{\alpha}(r, x)<+\infty .
\end{aligned}
$$

Let $\mathrm{g}$ be the function defined on $[0,+\infty[$ by

$$
g(s)=\frac{e^{s\left(\left|\left(\mu_{0}, \lambda_{0}\right)\right|-2 a\right)}}{\left(1+s+\left|\left(\mu_{0}, \lambda_{0}\right)\right|\right)^{d}}
$$

then $\mathrm{g}$ admits a minimum attained at

$$
s_{0}= \begin{cases}\frac{d}{\left|\left(\mu_{0}, \lambda_{0}\right)\right|-2 a}-1-\left|\left(\mu_{0}, \lambda_{0}\right)\right|, & \text { if } \frac{d}{\left|\left(\mu_{0}, \lambda_{0}\right)\right|-2 a}>1+\left|\left(\mu_{0}, \lambda_{0}\right)\right| \\ 0, & \text { if } \frac{d}{\left|\left(\mu_{0}, \lambda_{0}\right)\right|-2 a} \leqslant 1+\left|\left(\mu_{0}, \lambda_{0}\right)\right| .\end{cases}
$$

Consequently,

$$
\begin{aligned}
\int_{0}^{+\infty} \int_{\mathbb{R}}|f(r, x)| e^{2 a|(r, x)|} d v_{\alpha}(r, x) \\
\quad \leqslant \frac{1}{g\left(s_{0}\right)} \int_{0}^{+\infty} \int_{\mathbb{R}} \frac{|f(r, x)| e^{|(r, x)|\left|\left(\mu_{0}, \lambda_{0}\right)\right|}}{\left(1+|(r, x)|+\left|\left(\mu_{0}, \lambda_{0}\right)\right|\right)^{d}} d v_{\alpha}(r, x) \\
<+\infty
\end{aligned}
$$


On the other hand, from the relation (2.1) we deduce that for all $(r, x) \in[0,+\infty[\times \mathbb{R}$, the function

$$
(\mu, \lambda) \longmapsto j_{\alpha}(r \mu) e^{-i x \lambda}
$$

is analytic on $\mathbb{C}^{2}$ [7, even with respect to the first variable, and by the relation (2.3) we have

$$
\left|j_{\alpha}(r \mu) e^{-i \lambda x}\right| \leqslant e^{|(r, x)|(|\operatorname{Im}(\mu)|+|\operatorname{Im}(\lambda)|)} .
$$

From the relations (2.7), (3.2), and (3.3), it follows that the function $\widetilde{\mathscr{F}_{\alpha}}(\mathrm{f})$ is analytic on $\mathrm{B}_{\mathrm{a}}$, even with respect to the first variable.

Corollary 3.1. Let $f \in \mathrm{L}^{2}\left(\mathrm{~d} v_{\alpha}\right) ; f \neq 0$; and let $\mathrm{d}$ be a real number, $\mathrm{d} \geqslant 0$. If

$$
\iint_{\Gamma_{+}} \int_{0}^{+\infty} \int_{\mathbb{R}} \frac{|f(r, x)|\left|\mathscr{F}_{\alpha}(f)(\mu, \lambda)\right|}{(1+|(r, x)|+|\theta(\mu, \lambda)|)^{d}} e^{|(r, x)||\theta(\mu, \lambda)|} \mathrm{d} v_{\alpha}(r, x) d \widetilde{\gamma}_{\alpha}(\mu, \lambda)<+\infty .
$$

then for all real number $a$; $a>0$, we have

$$
v_{\alpha}\left(\left\{(r, x) \in \mathbb{R}^{2} \mid \widetilde{\mathscr{F}}_{\alpha}(f)(r, x) \neq 0 \quad \text { and } \quad|(r, x)|>a\right\}\right)>0 .
$$

Proof. From lemma 3.2, the function $f$ belongs to $L^{1}\left(d v_{\alpha}\right)$, and consequently the function $\widetilde{\mathscr{F}}_{\alpha}(f)$ is continuous on $\mathbb{R}^{2}$, even with respect to the first variable.

Then for all $a>0$, the set

$$
\left\{(r, x) \in \mathbb{R}^{2} \mid \widetilde{\mathscr{F}}_{\alpha}(f)(r, x) \neq 0 \text { and }|(r, x)|>a\right\},
$$

is on open subset of $\mathbb{R}^{2}$.

Assume that

$$
v_{\alpha}\left(\left\{(r, x) \in \mathbb{R}^{2} \mid \widetilde{\mathscr{F}}_{\alpha}(f)(r, x) \neq 0 \quad \text { and } \quad|(r, x)|>a\right\}\right)=0,
$$

then for all $(r, x) \in \mathbb{R}^{2} ;|(r, x)|>a$, we have $\widetilde{\mathscr{F}}_{\alpha}(f)(r, x)=0$.

Applying lemma 3.3 and analytic continuation, we deduce that $\widetilde{\mathscr{F}}_{\alpha}(\mathrm{f})$ vanishes on $\mathbb{R}^{2}$, and by theorem 2.1, it follows that $f=0$.

Lemma 3.4. Let $\mathrm{f} \in \mathrm{L}^{2}\left(\mathrm{~d} v_{\alpha}\right)$ and let $\mathrm{d}$ be a real number $\mathrm{d} \geqslant 0$. If

$$
\iint_{\Gamma_{+}} \int_{0}^{+\infty} \int_{\mathbb{R}} \frac{|f(r, x)|\left|\mathscr{F}_{\alpha}(f)(\mu, \lambda)\right|}{(1+|(r, x)|+|\theta(\mu, \lambda)|)^{d}} e^{|(r, x)||\theta(\mu, \lambda)|} \mathrm{d} v_{\alpha}(r, x) d \widetilde{\gamma}_{\alpha}(\mu, \lambda)<+\infty,
$$

then the function $\mathscr{W}_{\alpha}(\mathrm{f})$, belongs to $\mathrm{L}^{2}\left(\mathrm{dm}_{2}\right)$, where $\mathscr{W}_{\alpha}$ is the mapping defined by the relation (2.11).

Proof. From the hypothesis and the relations (2.5) and (2.6), we have

$$
\begin{aligned}
& \iint_{\Gamma_{+}} \int_{0}^{+\infty} \int_{\mathbb{R}} \frac{|f(r, x)|\left|\mathscr{F}_{\alpha}(f)(\mu, \lambda)\right|}{(1+|(r, x)|+|\theta(\mu, \lambda)|)^{\mathrm{d}}} e^{|(r, x)||\theta(\mu, \lambda)|} \mathrm{d} v_{\alpha}(r, x) \mathrm{d} \widetilde{\gamma}_{\alpha}(\mu, \lambda) \\
& \quad=\int_{0}^{+\infty} \int_{\mathbb{R}} \int_{0}^{+\infty} \int_{\mathbb{R}} \frac{|f(r, x)|\left|\widetilde{F}_{\alpha}(f)(\mu, \lambda)\right|}{(1+|(r, x)|+|(\mu, \lambda)|)^{\mathrm{d}}} e^{|(r, x) \|(\mu, \lambda)|} \mathrm{d} v_{\alpha}(r, x) \mathrm{dm}_{2}(\mu, \lambda)<+\infty .
\end{aligned}
$$


By the same way as inequality (3.2) of the lemma 3.3, there exists $b \in \mathbb{R}, b>0$, such that

$$
\int_{0}^{+\infty} \int_{\mathbb{R}}\left|\widetilde{\mathscr{F}}_{\alpha}(f)(\mu, \lambda)\right| e^{\mathfrak{b}|(\mu, \lambda)|} \mathrm{d} m_{2}(\mu, \lambda)<+\infty .
$$

Consequently, the function $\widetilde{\mathscr{F}}_{\alpha}(\mathrm{f})$ lies in $\mathrm{L}^{1}\left(\mathrm{~d} v_{\alpha}\right)$ and by theorem 2.1 we get

$$
f(r, x)=\int_{0}^{+\infty} \int_{\mathbb{R}} \widetilde{\mathscr{F}}_{\alpha}(f)(\mu, \lambda) j_{\alpha}(r \mu) e^{i \lambda x} \mathrm{~d} v_{\alpha}(\mu, \lambda) ; \quad \text { a.e. }
$$

In particular the function $f$ is bounded and

$$
\|f\|_{\infty, v_{\alpha}} \leqslant\left\|\widetilde{\mathscr{F}}_{\alpha}(f)\right\|_{1, v_{\alpha}} .
$$

Now, we have

$$
\begin{aligned}
\left|\mathscr{W}_{\alpha}(f)(r, x)\right| & \leqslant \frac{1}{2^{\alpha+\frac{1}{2}} \Gamma\left(\alpha+\frac{1}{2}\right)} \int_{r}^{+\infty}\left(t^{2}-r^{2}\right)^{\alpha-\frac{1}{2}}|f(t, x)| 2 t d t \\
& =\frac{r^{2 \alpha+1}}{2^{\alpha+\frac{1}{2}} \Gamma\left(\alpha+\frac{1}{2}\right)} \int_{1}^{+\infty}\left(u^{2}-1\right)^{\alpha-\frac{1}{2}}|f(r u, x)| 2 u d u .
\end{aligned}
$$

Using Minkowski's inequality for integrals [1], we get

$$
\begin{aligned}
& \left(\int_{0}^{+\infty} \int_{\mathbb{R}}\left|\mathscr{W}_{\alpha}(f)(r, x)\right|^{2} \mathrm{dm} m_{2}(r, x)\right)^{\frac{1}{2}} \\
& \leqslant \frac{1}{2^{\alpha+\frac{1}{2}} \Gamma\left(\alpha+\frac{1}{2}\right)}\left(\int_{0}^{+\infty} \int_{\mathbb{R}}\left(\int_{1}^{+\infty} r^{2 \alpha+1}\left(u^{2}-1\right)^{\alpha-\frac{1}{2}}|f(r u, x)| 2 u d u\right)^{2} \mathrm{dm}_{2}(r, x)\right)^{\frac{1}{2}} \\
& \leqslant \frac{1}{2^{\alpha+\frac{1}{2}} \Gamma\left(\alpha+\frac{1}{2}\right)} \int_{1}^{+\infty}\left(u^{2}-1\right)^{\alpha-\frac{1}{2}}\left(\int_{0}^{+\infty} \int_{\mathbb{R}} r^{4 \alpha+2}|f(r u, x)|^{2} d m_{2}(r, x)\right)^{\frac{1}{2}} 2 u d u \\
& =\frac{\Gamma(\alpha+1)^{\frac{1}{2}}}{2^{\frac{\alpha}{2}-\frac{3}{4}} \pi^{\frac{1}{4}} \Gamma\left(\alpha+\frac{1}{2}\right)}\left(\int_{1}^{+\infty}\left(u^{2}-1\right)^{\alpha-\frac{1}{2}} u^{-2 \alpha-\frac{1}{2}} d u\right)\left(\int_{0}^{+\infty} \int_{\mathbb{R}}|f(t, x)|^{2} t^{2 \alpha+1} d v_{\alpha}(t, x)\right)^{\frac{1}{2}} \\
& =\frac{\Gamma(\alpha+1)^{\frac{1}{2}}}{2^{\frac{\alpha}{2}-\frac{7}{4}} \pi^{\frac{1}{4}} \Gamma\left(\alpha+\frac{1}{2}\right)}\left(\int_{0}^{1}(1-s)^{\alpha-\frac{1}{2}} s^{\frac{9}{4}} \mathrm{~d} s\right)\left(\int_{0}^{+\infty} \int_{\mathbb{R}}|f(t, x)|^{2} \mathrm{t}^{2 \alpha+1} \mathrm{~d} v_{\alpha}(\mathrm{t}, x)\right)^{\frac{1}{2}} \\
& =\mathrm{C}_{\alpha}\left(\int_{0}^{+\infty} \int_{\mathbb{R}}|\mathrm{f}(\mathrm{t}, \mathrm{x})|^{2} \mathrm{t}^{2 \alpha+1} \mathrm{~d} v_{\alpha}(\mathrm{t}, \mathrm{x})\right)^{\frac{1}{2}}
\end{aligned}
$$

and by the relations (3.2) and (3.5), we get

$$
\begin{aligned}
\left(\int_{0}^{+\infty} \int_{\mathbb{R}}\left|\mathscr{W}_{\alpha}(f)(r, x)\right|^{2} \mathrm{dm}_{2}(\mathrm{r}, x)\right)^{\frac{1}{2}} & \leqslant M_{\alpha}\|f\|_{\infty, v_{\alpha}}^{\frac{1}{2}}\left(\int_{0}^{+\infty} \int_{\mathbb{R}}|f(t, x)| e^{2 a|(t, x)|} \mathrm{d} v_{\alpha}(t, x)\right)^{\frac{1}{2}} \\
& <+\infty
\end{aligned}
$$

Remark 3.1. Let $f$ be a function satisfying the hypothesis (3.1), then from the relations (3.2) and (3.4), we can prove that the function $f$ belongs to the Schwartz's space $S_{*}\left(\mathbb{R}^{2}\right)$. Since the Weyl transform $\mathscr{W}_{\alpha}$ is an isomorphism from $S_{*}\left(\mathbb{R}^{2}\right)$ onto itself, then the function $\mathscr{W}_{\alpha}(f)$ belongs to $S_{*}\left(\mathbb{R}^{2}\right)$, in particular $\mathscr{W}_{\alpha}(f) \in \mathrm{L}^{2}\left(\mathrm{dm}_{2}\right)$. 
Remark 3.2. Let $\sigma$ be a positive real number such that $\sigma+\sigma^{2}>d \geqslant 0$. Then, the function

$$
t \longmapsto \frac{e^{\sigma t}}{(1+t+\sigma)^{d}}
$$

is increasing on $[0,+\infty[$.

Theorem 3.5. Let $\mathrm{f} \in \mathrm{L}^{2}\left(\mathrm{~d} v_{\alpha}\right)$, and let $\mathrm{d}$ be a real number, $\mathrm{d} \geqslant 0$. If

$$
\iint_{\Gamma_{+}} \int_{0}^{+\infty} \int_{\mathbb{R}} \frac{|f(r, x)|\left|\mathscr{F}_{\alpha}(f)(\mu, \lambda)\right|}{(1+|(r, x)|+|\theta(\mu, \lambda)|)^{d}} e^{|(r, x)||\theta(\mu, \lambda)|} \mathrm{d} v_{\alpha}(r, x) d \widetilde{\gamma}_{\alpha}(\mu, \lambda)<+\infty,
$$

then

$$
\int_{0}^{+\infty} \int_{\mathbb{R}} \int_{0}^{+\infty} \int_{\mathbb{R}} \frac{\left|\mathscr{W}_{\alpha}(f)(r, x) \| \widetilde{\mathscr{F}}_{\alpha}(f)(\mu, \lambda)\right|}{(1+|(r, x)|+|(\mu, \lambda)|)^{\mathrm{d}}} e^{|(r, x) \|(\mu, \lambda)|} \mathrm{dm}_{2}(r, x) \mathrm{dm}_{2}(\mu, \lambda)<+\infty
$$

Proof. From the hypothesis and the relations (2.5) and (2.6), we have

$$
\int_{0}^{+\infty} \int_{\mathbb{R}} \int_{0}^{+\infty} \int_{\mathbb{R}} \frac{|f(r, x)|\left|\widetilde{\mathscr{F}}_{\alpha}(f)(\mu, \lambda)\right|}{(1+|(r, x)|+|(\mu, \lambda)|)^{\mathrm{d}}} e^{|(r, x)||(\mu, \lambda)|} \mathrm{d} v_{\alpha}(r, x) \mathrm{dm}_{2}(\mu, \lambda)<+\infty .
$$

i) If $d=0$, then by Fubini's theorem we have

$$
\begin{aligned}
& \int_{0}^{+\infty} \int_{\mathbb{R}} \int_{0}^{+\infty} \int_{\mathbb{R}}\left|\mathscr{W}_{\alpha}(f)(r, x) \| \widetilde{\mathscr{F}}_{\alpha}(f)(\mu, \lambda)\right| e^{|(r, x) \||(\mu, \lambda) \mid} \mathrm{dm}_{2}(r, x) \mathrm{dm}_{2}(\mu, \lambda) \\
& \quad \leqslant \int_{0}^{+\infty} \int_{\mathbb{R}}\left|\widetilde{\mathscr{F}}_{\alpha}(f)(\mu, \lambda)\right|\left(\int_{0}^{+\infty} \int_{\mathbb{R}}\left|\mathscr{W}_{\alpha}(f)(r, x)\right| e^{|(r, x)||(\mu, \lambda)|} \mathrm{dm}_{2}(r, x)\right) \mathrm{dm}_{2}(\mu, \lambda) \\
& \quad \leqslant \int_{0}^{+\infty} \int_{\mathbb{R}}\left|\widetilde{\mathscr{F}}_{\alpha}(f)(\mu, \lambda)\right|\left(\int_{0}^{+\infty} \int_{\mathbb{R}} \mathscr{W}_{\alpha}(|f|)(r, x) e^{|(r, x)||(\mu, \lambda)|} \mathrm{dm}_{2}(r, x)\right) \mathrm{dm}_{2}(\mu, \lambda) .
\end{aligned}
$$

Using the relation (2.10), we deduce that

$$
\int_{0}^{+\infty} \int_{\mathbb{R}} \mathscr{W}_{\alpha}(|f|)(r, x) e^{|(r, x) \|(\mu, \lambda)|} \mathrm{dm}_{2}(r, x)=\int_{0}^{+\infty} \int_{\mathbb{R}}|f(r, x)| \Re_{\alpha}\left(e^{|(., .)||(\mu, \lambda)|}\right)(r, x) d v_{\alpha}(r, x),
$$

but for all $(r, x) \in[0,+\infty[\times \mathbb{R}$

$$
\mathfrak{R}_{\alpha}\left(e^{|(, .,)|(\mu, \lambda) \mid}\right)(r, x) \leqslant e^{|(r, x) \|(\mu, \lambda)|} .
$$

Combining the relations (3.6), (3.7), (3.8), and (3.9), we get

$$
\begin{aligned}
\int_{0}^{+\infty} \int_{\mathbb{R}} & \int_{0}^{+\infty} \int_{\mathbb{R}}\left|\mathscr{W}_{\alpha}(\mathrm{f})(\mathrm{r}, \mathrm{x})\right|\left|\widetilde{\mathscr{F}}_{\alpha}(\mathrm{f})(\mu, \lambda)\right| e^{|(\mathrm{r}, \mathrm{x})||(\mu, \lambda)|} \mathrm{dm}_{2}(\mathrm{r}, \mathrm{x}) \mathrm{dm}_{2}(\mu, \lambda) \\
& \leqslant \int_{0}^{+\infty} \int_{\mathbb{R}}\left|\widetilde{\mathscr{F}}_{\alpha}(\mathrm{f})(\mu, \lambda)\right|\left(\int_{0}^{+\infty} \int_{\mathbb{R}}|\mathrm{f}(\mathrm{r}, \mathrm{x})| e^{|(r, x)||(\mu, \lambda)|} \mathrm{d} v_{\alpha}(\mathrm{r}, x)\right) \mathrm{dm}_{2}(\mu, \lambda) \\
& <+\infty .
\end{aligned}
$$


ii) If $d>0$, let

$$
B_{d}=\{(u, v) \in[0,+\infty[\times \mathbb{R}||(u, v) \mid \leqslant d\} .
$$

. By Fubini's theorem, we have

$$
\begin{aligned}
\iint_{B_{d}^{c}} \int_{0}^{+\infty} \int_{\mathbb{R}} \frac{\left|\widetilde{\mathscr{F}}_{\alpha}(f)(\mu, \lambda)\right|\left|\mathscr{W}_{\alpha}(f)(r, x)\right|}{(1+|(r, x)|+|(\mu, \lambda)|)^{\mathrm{d}}} e^{|(r, x)||(\mu, \lambda)|} \mathrm{dm}_{2}(\mathrm{r}, x) \mathrm{dm}_{2}(\mu, \lambda) \\
\quad \leqslant \iint_{B_{\mathrm{d}}^{c}}\left|\widetilde{\mathscr{F}}_{\alpha}(\mathrm{f})(\mu, \lambda)\right|\left(\int_{0}^{+\infty} \int_{\mathbb{R}} \mathscr{W}_{\alpha}(|\mathrm{f}|)(\mathrm{r}, \mathrm{x}) \frac{\mathrm{e}^{|(\mathrm{r}, \mathrm{x})||(\mu, \lambda)|}}{(1+|(\mathrm{r}, \mathrm{x})|+|(\mu, \lambda)|)^{\mathrm{d}}}\right. \\
\left.\quad \times \mathrm{dm}_{2}(\mathrm{r}, \mathrm{x})\right) \mathrm{dm}_{2}(\mu, \lambda),
\end{aligned}
$$

and by the relation (2.10), we get

$$
\begin{aligned}
& \iint_{B_{d}^{c}} \int_{0}^{+\infty} \int_{\mathbb{R}} \frac{\left|\widetilde{F}_{\alpha}(f)(\mu, \lambda)\right|\left|\mathscr{W}_{\alpha}(f)(r, x)\right|}{(1+|(r, x)|+|(\mu, \lambda)|)^{\mathrm{d}}} e^{|(r, x)||(\mu, \lambda)|} \mathrm{dm}_{2}(r, x) \mathrm{dm}_{2}(\mu, \lambda) \\
& \quad \leqslant \iint_{B_{d}^{c}}\left|\widetilde{\mathscr{F}}_{\alpha}(f)(\mu, \lambda)\right|\left(\int_{0}^{+\infty} \int_{\mathbb{R}}|f(r, x)| \Re_{\alpha}\left(\frac{e^{|(., .)||(\mu, \lambda)|}}{(1+|(., .)|+|(\mu, \lambda)|)^{\mathrm{d}}}\right)(r, x)\right. \\
& \left.\quad \times d v_{\alpha}(r, x)\right) \mathrm{dm}_{2}(\mu, \lambda) .
\end{aligned}
$$

However, by the relation (2.9) and remark 3.2 we have for all $(\mu, \lambda) \in B_{d}^{c}$

$$
\mathfrak{R}_{\alpha}\left(\frac{e^{|(., .)|(\mu, \lambda) \mid}}{(1+|(., .)|+|(\mu, \lambda)|)^{\mathrm{d}}}\right)(r, x) \leqslant \frac{e^{|(r, x)||(\mu, \lambda)|}}{(1+|(r, x)|+|(\mu, \lambda)|)^{\mathrm{d}}} .
$$

Combining the relations (3.10) and (3.11), we obtain

$$
\begin{aligned}
& \iint_{B_{d}^{c}} \int_{0}^{+\infty} \int_{\mathbb{R}} \frac{\left|\widetilde{\mathscr{F}}_{\alpha}(f)(\mu, \lambda)\right|\left|\mathscr{W}_{\alpha}(f)(r, x)\right|}{(1+|(r, x)|+|(\mu, \lambda)|)^{d}} e^{|(r, x)||(\mu, \lambda)|} \mathrm{dm}_{2}(r, x) \mathrm{dm}_{2}(\mu, \lambda) \\
& \left.\leqslant \iint_{B_{d}^{c}}\left|\widetilde{\mathscr{F}}_{\alpha}(f)(\mu, \lambda)\right|\left(\int_{0}^{+\infty} \int_{\mathbb{R}}|f(r, x)| \frac{e^{|(r, x)||(\mu, \lambda)|}}{(1+|(r, x)|+|(\mu, \lambda)|)^{d}}\right) d v_{\alpha}(r, x)\right) d m_{2}(\mu, \lambda) \\
& \leqslant \int_{0}^{+\infty} \int_{\mathbb{R}} \int_{0}^{+\infty} \int_{\mathbb{R}} \frac{\left|\widetilde{\mathscr{F}}_{\alpha}(\mathrm{f})(\mu, \lambda)\right||\mathrm{f}(\mathrm{r}, \mathrm{x})|}{(1+|(\mathrm{r}, x)|+|(\mu, \lambda)|)^{\mathrm{d}}} e^{|(\mathrm{r}, x)||(\mu, \lambda)|} \mathrm{d} v_{\alpha}(\mathrm{r}, x) \mathrm{dm}_{2}(\mu, \lambda)<+\infty . \\
& \cdot \iint_{B_{d}} \iint_{B_{d}^{c}} \frac{\left|\mathscr{W}_{\alpha}(f)(r, x)\right|\left|\widetilde{F}_{\alpha}(f)(\mu, \lambda)\right|}{(1+|(r, x)|+|(\mu, \lambda)|)^{d}} e^{|(r, x)||(\mu, \lambda)|} \mathrm{dm}_{2}(r, x) \mathrm{dm}_{2}(\mu, \lambda) \\
& \leqslant \iint_{B_{d}}\left|\widetilde{\mathscr{F}}_{\alpha}(f)(\mu, \lambda)\right|\left(\iint_{B_{d}^{c}} \mathscr{W}_{\alpha}(|f|)(r, x) \frac{e^{|(r, x)||(\mu, \lambda)|}}{(1+|(r, x)|+|(\mu, \lambda)|)^{d}} d m_{2}(r, x)\right) d m_{2}(\mu, \lambda) .
\end{aligned}
$$


But for $(\mu, \lambda) \in B_{d}$,

$$
\begin{aligned}
& \iint_{B_{\mathfrak{d}}^{c}} \mathscr{W}_{\alpha}(|f|)(r, x) \frac{e^{|(r, x)||(\mu, \lambda)|}}{(1+|(r, x)|+|(\mu, \lambda)|)^{d}} d m_{2}(r, x) \\
& \quad=\int_{0}^{+\infty} \int_{\mathbb{R}}|f(r, x)| \Re_{\alpha}\left(\frac{e^{\mid((.,)||(\mu, \lambda) \mid}}{(1+|(., .)|+|(\mu, \lambda)|)^{d}} \mathbf{1}_{B_{d}^{c}}\right)(r, x) d v_{\alpha}(r, x) \\
& \quad \leqslant \iint_{B_{d}^{c}}|f(r, x)| \frac{e^{d|(r, x)|}}{(1+|(r, x)|+d)^{d}} d v_{\alpha}(r, x) .
\end{aligned}
$$

Hence,

$$
\begin{aligned}
& \iint_{B_{d}} \iint_{B_{d}^{c}} \frac{\left|\mathscr{W}_{\alpha}(f)(r, x)\right|\left|\widetilde{\mathscr{F}}_{\alpha}(f)(\mu, \lambda)\right|}{(1+|(r, x)|+|(\mu, \lambda)|)^{d}} e^{|(r, x)||(\mu, \lambda)|} \mathrm{dm}_{2}(r, x) \mathrm{dm}_{2}(\mu, \lambda) \\
& \quad \leqslant\left(\iint_{B_{d}}\left|\widetilde{\mathscr{F}}_{\alpha}(f)(\mu, \lambda)\right| d m_{2}(\mu, \lambda)\right)\left(\iint_{B_{d}^{c}}|f(r, x)| \frac{e^{d|(r, x)|}}{(1+|(r, x)|+d) d} d v_{\alpha}(r, x)\right) .
\end{aligned}
$$

In virtue of the relation (2.8), we have

$$
\begin{aligned}
& \iint_{B_{d}} \iint_{B_{d}^{c}} \frac{\left|\mathscr{W}_{\alpha}(f)(r, x) \| \widetilde{\mathscr{F}}_{\alpha}(f)(\mu, \lambda)\right|}{(1+|(r, x)|+|(\mu, \lambda)|)^{d}} e^{|(r, x)||(\mu, \lambda)|} \mathrm{dm}_{2}(r, x) d m_{2}(\mu, \lambda) \\
\leqslant & \|f\|_{1, v_{\alpha}} m_{2}\left(B_{d}\right)\left(\iint_{B_{d}^{c}}|f(r, x)| \frac{e^{d|(r, x)|}}{(1+|(r, x)|+d)^{d}} d v_{\alpha}(r, x)\right) .
\end{aligned}
$$

On he other hand, from corollary 3.1 and the relation (3.6), there exists $\left(\mu_{0}, \lambda_{0}\right) \in\left[0,+\infty\left[\times \mathbb{R},\left|\left(\mu_{0}, \lambda_{0}\right)\right|>\right.\right.$ $\mathrm{d}, \widetilde{\mathscr{F}}_{\alpha}(f)\left(\mu_{0}, \lambda_{0}\right) \neq 0$, and

$$
\iint_{B_{d}^{c}}|f(r, x)| \frac{e^{\left|\left(\mu_{0}, \lambda_{0}\right)\right||(r, x)|}}{\left(1+|(r, x)|+\left|\left(\mu_{0}, \lambda_{0}\right)\right|\right)^{d}} d v_{\alpha}(r, x)<+\infty,
$$

so, by remark 3.2 ,

$$
\begin{aligned}
\iint_{B_{d}^{c}}|f(r, x)| & \frac{e^{d|(r, x)|}}{(1+|(r, x)|+d)^{d}} d v_{\alpha}(r, x) \\
& \leqslant \iint_{B_{d}^{c}}|f(r, x)| \frac{e^{\left|\left(\mu_{0}, \lambda_{0}\right)\right||(r, x)|}}{\left(1+|(r, x)|+\left|\left(\mu_{0}, \lambda_{0}\right)\right|\right)^{d}} d v_{\alpha}(r, x) \\
& <+\infty .
\end{aligned}
$$

The relations (3.12), (3.13), and (3.14) imply that

$$
\iint_{B_{d}} \iint_{B_{d}^{c}} \frac{\left|\mathscr{W}_{\alpha}(f)(r, x)\right|\left|\widetilde{F}_{\alpha}(f)(\mu, \lambda)\right|}{(1+|(r, x)|+|(\mu, \lambda)|)^{d}} e^{|(r, x)||(\mu, \lambda)|} \mathrm{dm}_{2}(r, x) \mathrm{dm}_{2}(\mu, \lambda)<+\infty .
$$


Finally

$$
\begin{aligned}
& \cdot \iint_{B_{d}} \iint_{B_{d}} \frac{\left|\mathscr{W}_{\alpha}(f)(r, x)\right|\left|\widetilde{\mathscr{F}}_{\alpha}(f)(\mu, \lambda)\right|}{(1+|(r, x)|+|(\mu, \lambda)|)^{d}} e^{|(r, x) \|(\mu, \lambda)|} \mathrm{dm}_{2}(r, x) \mathrm{dm}_{2}(\mu, \lambda) \\
& \quad \leqslant e^{d^{2}}\left(\iint_{B_{d}}\left|\widetilde{\mathscr{F}}_{\alpha}(f)(\mu, \lambda)\right| d m_{2}(\mu, \lambda)\right)\left(\iint_{B_{d}}\left|\mathscr{W}_{\alpha}(f)(r, x)\right| \mathrm{dm}_{2}(r, x)\right) \\
& \quad \leqslant e^{d^{2}} m_{2}\left(B_{d}\right)\left\|\mathscr{F}_{\alpha}(f)\right\|_{\infty, \gamma_{\alpha}}\left\|\mathscr{W}_{\alpha}(f)\right\|_{1, m_{2}},
\end{aligned}
$$

and therefore by the relations (2.8) and (2.12), we deduce that

$$
\begin{aligned}
\iint_{B_{d}} \iint_{B_{d}} \frac{\left|\mathscr{W}_{\alpha}(f)(r, x)\right|\left|\widetilde{\mathscr{F}}_{\alpha}(f)(\mu, \lambda)\right|}{(1+|(r, x)|+|(\mu, \lambda)|)^{d}} e^{|(r, x) \|(\mu, \lambda)|} \mathrm{dm}_{2}(r, x) \mathrm{dm}_{2}(\mu, \lambda) \\
\quad \leqslant e^{d^{2}} m_{2}\left(B_{d}\right)\|f\|_{1, v_{\alpha}}^{2} \\
\quad<+\infty
\end{aligned}
$$

and the proof of theorem 3.5 is complete.

Theorem 3.6 (Beurling-Hörmander for $\left.\mathscr{R}_{\alpha}\right)$. Let $\mathrm{f} \in \mathrm{L}^{2}\left(\mathrm{~d} v_{\alpha}\right)$, and let $\mathrm{d}$ be a real number, $\mathrm{d} \geqslant 0$.

If

$$
\iint_{\Gamma_{+}} \int_{0}^{+\infty} \int_{\mathbb{R}} \frac{|f(r, x)|\left|\mathscr{F}_{\alpha}(f)(\mu, \lambda)\right|}{(1+|(r, x)|+|\theta(\mu, \lambda)|)^{d}} e^{|(r, x)||\theta(\mu, \lambda)|} d v_{\alpha}(r, x) d \widetilde{\gamma}_{\alpha}(\mu, \lambda)<+\infty
$$

Then

i) For $\mathrm{d} \leqslant 2, \mathrm{f}=0$.

ii) For $\mathrm{d}>2$, there exist a positive constant a and a polynomial $\mathrm{P}$, even with respect to the first variable, such that

with $\operatorname{degree}(\mathrm{P})<\frac{\mathrm{d}}{2}-1$.

$$
f(r, x)=P(r, x) e^{-a\left(r^{2}+x^{2}\right)},
$$

Proof. Let $\mathrm{f} \in \mathrm{L}^{2}\left(\mathrm{~d} \mathrm{v}_{\alpha}\right)$, satisfying the hypothesis.

From proposition 2.2 lemma 3.2 and lemma 3.4. we deduce that the function $\mathscr{W}_{\alpha}(\mathrm{f})$ belongs to the space $L^{1}\left(d m_{2}\right) \cap L^{2}\left(d m_{2}\right)$ and that

$$
\widetilde{\mathscr{F}}_{\alpha}(f)=\Lambda_{2} \circ \mathscr{W}_{\alpha}(f)
$$

Thus from theorem 3.5, we get

$$
\int_{0}^{+\infty} \int_{\mathbb{R}} \int_{0}^{+\infty} \int_{\mathbb{R}} \frac{\left|\mathscr{W}_{\alpha}(f)(r, x)\right|\left|\Lambda_{2}\left(\mathscr{W}_{\alpha}(f)\right)(\mu, \lambda)\right| e^{|(r, x)||(\mu, \lambda)|}}{(1+|(r, x)|+|(\mu, \lambda)|)^{\mathrm{d}}} \mathrm{dm}_{2}(\mathrm{r}, x) \mathrm{dm}_{2}(\mu, \lambda)<+\infty .
$$

Applying theorem 3.1, when $f$ is replaced by $\mathscr{W}_{\alpha}(f)$, we deduce that

If $\mathrm{d} \leqslant 2, \mathscr{W}_{\alpha}(\mathrm{f})=0$, and by remark 2.1, $\mathrm{f}=0$. 
If $d>2$, then there exist $a>0$ and a polynomial $Q$ even with respect to the first variable such that

$$
\mathscr{W}_{\alpha}(f)(r, x)=Q(r, x) e^{-a\left(r^{2}+x^{2}\right)}=\sum_{2 p+q \leqslant m} a_{p, q} r^{2 p} \chi^{q} e^{-a\left(r^{2}+x^{2}\right)} .
$$

In particular, the function $\mathscr{W}_{\alpha}(f)$ belongs to the space $S_{*}\left(\mathbb{R}^{2}\right)$. From remark 2.1, the function $f$ belongs to $S_{*}\left(\mathbb{R}^{2}\right)$ and from the relation (2.14), we get

$$
\begin{aligned}
f(r, x) & =\mathscr{H}_{-\alpha-\frac{1}{2}}\left(\mathrm{Q}(\mathrm{t}, \mathrm{y}) \mathrm{e}^{-\mathrm{a}\left(\mathrm{t}^{2}+\mathrm{y}^{2}\right)}\right)(\mathrm{r}, \mathrm{x}) \\
& =(-1)^{\left[\alpha+\frac{1}{2}\right]+1} \mathscr{H}_{\left[\alpha+\frac{1}{2}\right]-\alpha+\frac{1}{2}}\left(\left(\frac{\partial}{\partial \mathrm{t}^{2}}\right)^{\left[\alpha+\frac{1}{2}\right]+1}\left(\mathrm{P}(\mathrm{t}, \mathrm{y}) \mathrm{e}^{-\mathrm{a}\left(\mathrm{t}^{2}+\mathrm{y}^{2}\right)}\right)\right)(\mathrm{r}, \mathrm{x}) \\
& =\sum_{2 \mathrm{p}+\mathrm{q} \leqslant \mathrm{m}} \mathrm{a}_{\mathrm{p}, \mathrm{q}}(-1)^{\left[\alpha+\frac{1}{2}\right]+1} \mathscr{H}_{\left[\alpha+\frac{1}{2}\right]-\alpha+\frac{1}{2}}\left(\left(\frac{\partial}{\partial \mathrm{t}^{2}}\right)^{\left[\alpha+\frac{1}{2}\right]+1}\left(\mathrm{t}^{2 \mathfrak{p}} \mathrm{y}^{\mathrm{q}} \mathrm{e}^{-\mathrm{a}\left(\mathrm{t}^{2}+\mathrm{y}^{2}\right)}\right)\right)(\mathrm{r}, \mathrm{x}) .
\end{aligned}
$$

However, for all $k \in \mathbb{N}$,

$$
\left(\frac{\partial}{\partial t^{2}}\right)^{k}\left(t^{2 p} y^{q} e^{-a\left(t^{2}+y^{2}\right)}\right)=\left(\sum_{j=0}^{\min (\mathfrak{p}, k)} C_{k}^{j} \frac{2^{j} p !}{(p-j) !}(-2 a)^{k-j} t^{2(p-j)}\right) y^{q} e^{-a\left(t^{2}+y^{2}\right)},
$$

and for all $\sigma \in \mathbb{R}, \sigma>0$,

$$
\mathscr{H}_{\sigma}\left(\mathrm{t}^{2 \mathrm{p}} \mathrm{y}^{\mathrm{q}} \mathrm{e}^{-\mathrm{a}\left(\mathrm{t}^{2}+\mathrm{y}^{2}\right)}\right)(\mathrm{r}, \mathrm{x})=\frac{1}{2^{\sigma} \Gamma(\sigma)}\left(\sum_{j=0}^{\mathrm{p}} \mathrm{C}_{\mathrm{p}}^{j} \frac{\Gamma(\sigma+\mathrm{p}-\mathrm{j})}{\mathrm{a}^{\sigma+p-j}} \mathrm{r}^{2 j}\right) \mathrm{x}^{\mathrm{q}} \mathrm{e}^{-\mathrm{a}\left(\mathrm{r}^{2}+\mathrm{x}^{2}\right)} .
$$

Combining the relations (3.15), (3.16) and (3.17), we deduce that

$$
f(r, x)=P(r, x) e^{-a\left(r^{2}+x^{2}\right)} .
$$

Where $\mathrm{P}$ is a polynomial, even with respect to the first variable and $\operatorname{degree}(\mathrm{P})=\operatorname{degree}(\mathrm{Q})$.

\section{Applications of Beurling-Hörmander theorem}

In this section, we shall deduce from the precedent Beurling-Hörmander theorem two most important uncertainty principles for the Fourier transform $\mathscr{F}_{\alpha}$, that are the Gelfand-Shilov and the Cowling-Price theorems.

Lemma 4.1. Let $\mathrm{P}$ be a polynomial on $\mathbb{R}^{2}, \mathrm{P} \neq 0$, with degree $(\mathrm{P})=\mathrm{m}$. Then there exist two positive constants $\mathrm{A}$ and $\mathrm{C}$ such that

$$
\forall t \geqslant A, \quad p(t)=\int_{0}^{2 \pi} \mid P\left(t \cos (\theta), t \sin (\theta) \mid d \theta \geqslant C t^{m} .\right.
$$


Proof. Let $\mathrm{P}$ be a polynomial on $\mathbb{R}^{2}, \mathrm{P} \neq 0$ and with $\operatorname{degree}(\mathrm{P})=\mathrm{m}$. We have

$$
p(t)=\int_{0}^{2 \pi}\left|\sum_{j=0}^{m} a_{j}(\theta) t^{j}\right| d \theta
$$

where the functions $a_{j}, 0 \leqslant j \leqslant m$, are continuous on $[0,2 \pi]$. It's clear that the function $p$ is continuous on $[0,+\infty[$, and by dominate convergence theorem's, we have

$$
p(t) \sim C_{m} t^{m} \quad(t \longrightarrow+\infty),
$$

where $C_{m}=\int_{0}^{2 \pi}\left|a_{m}(\theta)\right| d \theta>0$.

Now the relation (4.1) involves that there exists $A>0$ such that

$$
\forall t \geqslant A, p(t) \geqslant \frac{C_{m}}{2} t^{m}
$$

Theorem 4.2 (Gelfand-Shilov for $\mathscr{R}_{\alpha}$ ). Let $\mathrm{p}, \mathrm{q}$ be two conjugate exponents, $\left.\mathrm{p}, \mathrm{q} \in\right] 1,+\infty[$. Let $\xi, \eta$ be non negative real numbers such that $\xi \eta \geqslant 1$. Let $\mathrm{f}$ be a measurable function on $\mathbb{R}^{2}$, even with respect to the first variable, such that $\mathrm{f} \in \mathrm{L}^{2}\left(\mathrm{~d} v_{\alpha}\right)$.

If

$$
\int_{0}^{+\infty} \int_{\mathbb{R}} \frac{|f(r, x)| e^{\frac{\xi^{p}|(r, x)|^{p}}{p}}}{(1+|(r, x)|)^{d}} d v_{\alpha}(r, x)<+\infty
$$

and

$$
\iint_{\Gamma_{+}} \frac{\left|\mathscr{F}_{\alpha}(f)(\mu, \lambda)\right| e^{\frac{\eta^{\mathrm{q}}|\theta(\mu, \lambda)| \mathrm{q}}{\mathrm{q}}}}{(1+|\theta(\mu, \lambda)|)^{\mathrm{d}}} \mathrm{d} \widetilde{\gamma}_{\alpha}(\mu, \lambda)<+\infty ; \mathrm{d} \geqslant 0 .
$$

Then

i) For $\mathrm{d} \leqslant 1, \mathrm{f}=0$.

ii) For d $>1$, we have

a) $f=0$ for $\xi \eta>1$.

b) $f=0$ for $\xi \eta=1$, and $p \neq 2$.

c) $f(r, x)=P(r, x) e^{-a\left(r^{2}+x^{2}\right)}$ for $\xi \eta=1$ and $p=q=2$,

where $\mathrm{a}>0$ and $\mathrm{P}$ is a polynomial on $\mathbb{R}^{2}$ even with respect to the first variable, with degree $(\mathrm{P})<$ $\mathrm{d}-1$.

Proof. Let $\mathrm{f}$ be a function satisfying the hypothesis. Since $\xi \eta \geqslant 1$, and by a convexity argument, 
we have

$$
\begin{aligned}
& \iint_{\Gamma_{+}} \int_{0}^{+\infty} \int_{\mathbb{R}} \frac{|f(r, x)|\left|\mathscr{F}_{\alpha}(f)(\mu, \lambda)\right|}{(1+|(r, x)|+|\theta(\mu, \lambda)|)^{2 d}} e^{|(r, x)||\theta(\mu, \lambda)|} \mathrm{d} v_{\alpha}(r, x) d \widetilde{\gamma}_{\alpha}(\mu, \lambda) \\
& \leqslant \iint_{\Gamma_{+}} \int_{0}^{+\infty} \int_{\mathbb{R}} \frac{|f(r, x)|\left|\mathscr{F}_{\alpha}(f)(\mu, \lambda)\right|}{(1+|(r, x)|)^{\mathrm{d}}(1+|\theta(\mu, \lambda)|)^{\mathrm{d}}} e^{\xi \eta|(r, x) \| \theta(\mu, \lambda)|} \mathrm{d} v_{\alpha}(\mathrm{r}, x) \mathrm{d} \widetilde{\gamma}_{\alpha}(\mu, \lambda) \\
& \leqslant\left(\iint_{\Gamma_{+}} \frac{\left|\mathscr{F}_{\alpha}(f)(\mu, \lambda)\right|}{(1+|\theta(\mu, \lambda)|)^{\mathrm{d}}} e^{\frac{\eta^{\mathrm{q}}|\theta(\mu, \lambda)| \mathrm{q}}{q}} \mathrm{~d} \widetilde{\gamma}_{\alpha}(\mu, \lambda)\right) \\
& \times\left(\int_{0}^{+\infty} \int_{\mathbb{R}} \frac{|\mathrm{f}(\mathrm{r}, x)|}{(1+|(r, x)|)^{\mathrm{d}}} e^{\frac{\xi^{p}|(r, x)|^{p}}{p}} \mathrm{~d} v_{\alpha}(r, x)\right) \\
& <+\infty \text {. }
\end{aligned}
$$

Then from the Beurling-Hörmander theorem, we deduce that

i) For $d \leqslant 1, f=0$.

ii) For $d>1$, there exist a positive constant $a$, and a polynomial $\mathrm{P}$ on $\mathbb{R}^{2}$, even with respect to the first variable such that

$$
f(r, x)=P(r, x) e^{-a\left(r^{2}+x^{2}\right)},
$$

with degree $(\mathrm{P})<\mathrm{d}-1$, and by a standard calculus, we obtain

$$
\widetilde{\mathscr{F}}_{\alpha}(f)(\mu, \lambda)=Q(\mu, \lambda) e^{-\frac{1}{4 a}\left(\mu^{2}+\lambda^{2}\right)},
$$

where $\mathrm{Q}$ is a polynomial on $\mathbb{R}^{2}$, even with respect to the first variable, with $\operatorname{degree}(\mathrm{P})=\operatorname{degree}(\mathrm{Q})$. On the other hand, from the relations (2.5), (2.6), (4.2), (4.3) and (4.4), we get

$$
\begin{aligned}
\int_{0}^{+\infty} \int_{\mathbb{R}} \int_{0}^{+\infty} \int_{\mathbb{R}} \frac{|\mathrm{P}(\mathrm{r}, x)||\mathrm{Q}(\mu, \lambda)|}{(1+|(\mathrm{r}, x)|)^{\mathrm{d}}(1+|(\mu, \lambda)|)^{\mathrm{d}}} e^{\xi \eta \eta(r, x)||(\mu, \lambda) \mid-\mathrm{a}\left(\mathrm{r}^{2}+\mathrm{x}^{2}\right)} \\
\times \mathrm{e}^{-\frac{1}{4 a}\left(\mu^{2}+\lambda^{2}\right)} \mathrm{d} v_{\alpha}(\mathrm{r}, x) \mathrm{d} \mu \mathrm{d} \lambda<+\infty
\end{aligned}
$$

so

$$
\int_{0}^{+\infty} \int_{0}^{+\infty} \frac{\varphi(t)}{(1+t)^{d}} \frac{\psi(\rho)}{(1+\rho)^{d}} e^{\xi \eta \eta t \rho} e^{-a t^{2}} e^{-\frac{1}{4 a} \rho^{2}} t^{2 \alpha+2} \rho d t d \rho<+\infty
$$

where

$$
\varphi(t)=\int_{0}^{2 \pi}|\mathrm{P}(\mathrm{t} \cos (\theta), \mathrm{t} \sin (\theta))||\cos (\theta)|^{2 \alpha+1} \mathrm{~d} \theta
$$

and

$$
\psi(\rho)=\int_{0}^{2 \pi}|\mathrm{Q}(\rho \cos (\theta), \rho \sin (\theta))| \mathrm{d} \theta .
$$

. Suppose that $\xi \eta>1$. If $f \neq 0$, then each of the polynomials $P$ and $Q$ is not identically zero, let $\mathrm{m}=\operatorname{degree}(\mathrm{P})=\operatorname{degree}(\mathrm{Q})$.

From lemma 4.1, there exist two positive constants $A$ and $C$ such that

$$
\forall t \geqslant A, \quad \varphi(t) \geqslant C t^{m}
$$


and

$$
\forall \rho \geqslant A, \quad \psi(\rho) \geqslant C \rho^{m} .
$$

Then, the inequality (4.5) leads to

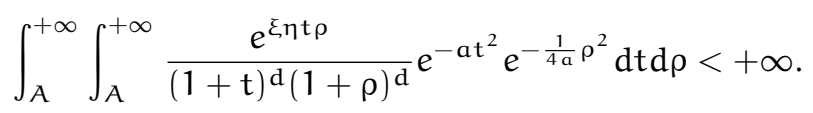

Let $\varepsilon>0$, such that $\xi \eta-\varepsilon=\sigma>1$. The relation (4.6) implies that

$$
\int_{A}^{+\infty} \int_{A}^{+\infty} \frac{e^{\varepsilon t \rho}}{(1+t)^{d}(1+\rho)^{d}} e^{\sigma t \rho} e^{-a t^{2}} e^{-\frac{1}{4 a} \rho^{2}} d t d \rho<+\infty .
$$

However, for all $t \geqslant A \geqslant \frac{d}{\varepsilon}$ and $\rho \geqslant A$, we have

$$
\frac{e^{\varepsilon \rho t}}{(1+t)^{d}(1+\rho)^{d}} \geqslant \frac{e^{\varepsilon A^{2}}}{(1+A)^{2 d}},
$$

and by the relation (4.7) it follows that

$$
\int_{A}^{+\infty} \int_{A}^{+\infty} e^{\sigma t \rho} e^{-a t^{2}} e^{-\frac{1}{4 a} \rho^{2}} d t d \rho<+\infty
$$

Let $F(t)=\int_{A}^{+\infty} e^{\sigma \rho t-\frac{1}{4 a} \rho^{2}} d \rho$, then $F$ can be written

$$
F(t)=e^{a \sigma^{2} t^{2}}\left(\int_{A}^{+\infty} e^{-\frac{1}{4 a} \rho^{2}} d \rho+2 a \sigma e^{-\frac{A^{2}}{4 a}} \int_{0}^{t} e^{A \sigma s-a \sigma^{2} s^{2}} d s\right),
$$

in particular

$$
F(t) \geqslant e^{a \sigma^{2} t^{2}} \int_{A}^{+\infty} e^{-\frac{1}{4 a} \rho^{2}} d \rho .
$$

Thus

$$
\begin{aligned}
\int_{A}^{+\infty} \int_{A}^{+\infty} e^{\sigma t \rho} e^{-a t^{2}} e^{-\frac{1}{4 a} \rho^{2}} d t d \rho & =\int_{A}^{+\infty} e^{-a t^{2}} F(t) d t \\
& \geqslant \int_{A}^{+\infty} e^{-\frac{1}{4 a} \rho^{2}} d \rho \int_{A}^{+\infty} e^{a\left(\sigma^{2}-1\right) t^{2}} d t=+\infty
\end{aligned}
$$

because $\sigma>1$. This contradics the relation (4.8) and shows that $f=0$.

. Suppose that $\xi \eta=1$ and $p \neq 2$. In this case we have $p>2$ or $q>2$. Suppose that $q>2$, then from the second hypothesis and the relation (4.4), we have

$$
\int_{0}^{+\infty} \frac{\psi(\rho) e^{-\frac{\rho^{2}}{4 a}} e^{\frac{\eta^{q} \rho^{q}}{q}}}{(1+\rho)^{d}} \rho d \rho<+\infty .
$$

If $f \neq 0$, then the polynomial $Q$ is not identically zero, and by lemma 4.1 and the relation (4.9), it follows that

$$
\int_{0}^{+\infty} \frac{e^{-\frac{\rho^{2}}{4 a}} e^{\frac{\eta^{q} \rho^{q}}{q}}}{(1+\rho)^{d}} d \rho<+\infty,
$$


which is impossible because $q>2$.

The proof of theorem 4.2 is complete.

Theorem 4.3 (Cowling-Price for $\mathscr{R}_{\alpha}$ ). Let $\xi, \eta, \omega_{1}, \omega_{2}$ be non negative real numbers such that $\xi \eta \geqslant \frac{1}{4}$. Let $\mathrm{p}, \mathrm{q}$ be two exponents, $\mathrm{p}, \mathrm{q} \in[1,+\infty]$, and let $\mathrm{f}$ be a measurable function on $\mathbb{R}^{2}$, even with respect to the first variable such that $\mathrm{f} \in \mathrm{L}^{2}\left(\mathrm{~d} v_{\alpha}\right)$.

If

$$
\left\|\frac{e^{\xi|(. . .)|^{2}}}{(1+|(., .)|)^{\omega_{1}}} f\right\|_{p, v_{\alpha}}<+\infty
$$

and

$$
\left\|\frac{e^{\eta|\theta(. . .)|^{2}}}{(1+|\theta(., .)|)^{\omega_{2}}} \mathscr{F}_{\alpha}(f)\right\|_{\mathbf{q}, \tilde{\gamma}_{\alpha}}<+\infty
$$

then

i) For $\xi \eta>\frac{1}{4}, f=0$.

ii) For $\xi \eta=\frac{1}{4}$, there exist a positive constant a and a polynomial $\mathrm{P}$ on $\mathbb{R}^{2}$, even with respect to the first variable, such that

$$
f(r, x)=P(r, x) e^{-a\left(r^{2}+x^{2}\right)}
$$

Proof. Let $\mathrm{p}^{\prime}$ and $\mathrm{q}^{\prime}$ be the conjugate exponents of $\mathrm{p}$ respectively $\mathrm{q}$. Let us pick $\mathrm{d}_{1}, \mathrm{~d}_{2} \in \mathbb{R}$, such that $d_{1}>2 \alpha+3$ and $d_{2}>2$. Finally, let $d$ be a positive real number such that $d>$ $\max \left(\omega_{1}+\frac{d_{1}}{p^{\prime}}, \omega_{2}+\frac{d_{2}}{q^{\prime}}, 1\right)$.

From Hölder's inequality and the relations (4.10) and (4.11), we deduce that

$$
\int_{0}^{+\infty} \int_{\mathbb{R}} \frac{|f(r, x)| e^{\xi|(r, x)|^{2}}}{(1+|(r, x)|)^{\omega_{1}+\frac{d_{1}}{p^{\prime}}}} d v_{\alpha}(r, x)<+\infty,
$$

and

$$
\iint_{\Gamma_{+}} \frac{\left|\mathscr{F}_{\alpha}(f)(\mu, \lambda)\right| e^{\mathfrak{\eta}|\theta(\mu, \lambda)|^{2}}}{(1+|\theta(\mu, \lambda)|)^{\omega_{2}+\frac{d_{2}}{q^{\prime}}}} \mathrm{d} \widetilde{\gamma}_{\alpha}(\mu, \lambda)<+\infty .
$$

Consequently we have

$$
\int_{0}^{+\infty} \int_{\mathbb{R}} \frac{|f(r, x)| e^{\xi|(r, x)|^{2}}}{(1+|(r, x)|)^{d}} d v_{\alpha}(r, x)<+\infty,
$$

and

$$
\iint_{\Gamma_{+}} \frac{\left|\mathscr{F}_{\alpha}(f)(\mu, \lambda)\right| e^{\mathfrak{\eta}|\theta(\mu, \lambda)|^{2}}}{(1+|\theta(\mu, \lambda)|)^{\mathrm{d}}} \mathrm{d} \widetilde{\gamma}_{\alpha}(\mu, \lambda)<+\infty .
$$

Then, the desired result follows from theorem 4.2

Remark 4.1. The Hardy's theorem is a special case of theorem 4.3 when $p=q=+\infty$.

Received: July 2010. Revised: August 2010. 


\section{References}

[1] G. Andrews, R. Askey and R. Roy, Special Functions, Cambridge University Press, New-York 1999.

[2] C. Baccar, N. B. Hamadi and L. T. Rachdi, Inversion formulas for the Riemann-Liouville transform and its dual associated with singular partial differential operators, Int. J. Math. Math. Sci., 2006 (2006), pp 1-26.

[3] S. Ben Farah and K. Mokni, Uncertainty Principle and the (Lp, Lq) version of Morgans theorem on some groups, Russ. J. Math. Phys., 10 No. 3 (2003), pp 245-260.

[4] A. Beurling, The collected works of Arne Beurling, Birkhuser., Vol.1-2, Boston 1989.

[5] A. Bonami, B. Demange, and P. Jaming, Hermite functions and uncertainty priciples for the Fourier and the widowed Fourier transforms, Rev. Mat. Iberoamericana., 19 (2003), pp 23-55.

[6] L. Bouattour and K. Trimèche, An analogue of the Beurling-Hörmander's theorem for the Chébli-Trimèche transform, Glob. J. Pure Appl. Math., 1 No. 3 (2005), pp 342-357.

[7] B. Chabat, Introduction à l'analyse complexe, Edition Mir., Vol.2, Moscou 1985.

[8] M.G. Cowling and J. F. Price, Generalizations of Heisenbergs inequality in Harmonic analysis, (Cortona, 1982), Lecture Notes in Math., 992 (1983), pp 443-449.

[9] A. Erdely and all, Asymptotic expansions, Dover publications, New-York 1956.

[10] A. Erdely and all, Tables of integral transforms, Mc Graw-Hill Book Compagny., Vol.2, New York 1954.

[11] G. B. Folland, Real Analysis Modern Techniques and Their Applications, Pure and Applied Mathematics, John Wiley and Sons., New York 1984.

[12] G. B. Folland and A. Sitaram, The uncertainty principle: a mathematical survey, J. Fourier Anal. Appl., 3 (1997), pp 207-238.

[13] I.M. Gelfand and G.E. Shilov, Fourier transforms of rapidly increasing functions and questions of uniqueness of the solution of Cauchy's problem, Uspekhi Mat. Nauk., 8 (1953), pp 3-54.

[14] G. H. Hardy, A theorem concerning Fourier transform, J. London. Math. Soc., 8 (1933), pp $227-231$.

[15] V. Havin and B. Jöricke, An uncertainty principle in harmonic analysis, Springer Verlag., Berlin 1994.

[16] L. Hörmander, A uniqueness theorem of Beurling for Fourier transform pairs, Ark. Mat., 29 (1991), pp 237-240. 
[17] L. Kamoun and K. Trimèche, An Analogue of Beurling-Hörmander's Theorem Associated with Partial Differential Operators, Meditter. J. Math., 2 (2005), pp 243-258.

[18] N. N. Lebedev, Special Functions and their applications, Dover publications., New-York 1972.

[19] G. W. Morgan, A note on Fourier transforms, J. London. Math. Soc., 9 (1934), pp 178-192.

[20] S. Omri and L. T. Rachdi, An $\mathrm{L}^{\mathrm{p}}$ - $\mathrm{L}^{\mathrm{q}}$ version of Morgan's theorem associated with RiemannLiouville transform, Int. J. Math. Anal., 1 No. 17 (2007), pp 805-824.

[21] S. Omri and L. T. Rachdi, Heisenberg-Pauli-Weyl uncertainty principle for the RiemannLiouville Operator, J. Ineq. Pure and Appl. Math., 9 (2008), Iss. 3, Art 88.

[22] K. Trimèche, Beurling-Hörmander's theorem for the Dunkl transform, Glob. J. Pure Appl. Math., 2 No. 3 (2006), pp 181-196.

[23] K. Trimèche, Inversion of the Lions translation operator using genaralized wavelets, Appl. Comput. Harmonic Anal., 4 (1997), pp 97-112.

[24] K. Trimèche, Transformation intégrale de Weyl et théorème de Paley-Wienner associés à un opérateur différentiel singulier sur $(0,+\infty), J$. Math. Pures Appl., 60 (1981), pp 51-98.

[25] G. N. Watson, A treatise on the theory of Bessel functions, Cambridge univ. Press., 2nd ed, Cambridge 1959.

[26] S.B. Yakubovich, Uncertainty principles for the Kontorovich-Lebedev transform, Math. Modell. Anal., 13 No. 2 (2008), pp 289-302. 